\title{
Synthetic Siglec-9 agonists inhibit neutrophil activation associated with COVID-19
}

\section{Authors.}

Corleone S. Delaveris, ${ }^{1,2}$ Aaron J. Wilk, ${ }^{3,4,5}$ Nicholas M. Riley, ${ }^{1}$ Jessica C. Stark, ${ }^{1}$ Samuel S. Yang, ${ }^{6}$ Angela J. Rogers, ${ }^{5}$ Thanmayi Ranganath, ${ }^{5}$ Kari C. Nadeau,,${ }^{5,7}$ the Stanford COVID-19 Biobank, ${ }^{8}$ Catherine A. Blish, ${ }^{5,9}$ Carolyn R. Bertozzi ${ }^{1,2,10}$

\section{Author Affiliations.}

${ }^{1}$ Department of Chemistry, Stanford University, Stanford CA, 94305

${ }^{2} \mathrm{ChEM}-\mathrm{H}$, Stanford University, Stanford, CA 94305

${ }^{3}$ Stanford Medical Scientist Training Program, Stanford, CA 94305

${ }^{4}$ Stanford Immunology Program, Stanford University, Stanford, CA 94305

${ }^{5}$ Department of Medicine, Stanford University, Stanford, CA 94305

${ }^{6}$ Department of Emergency Medicine, Stanford University, Stanford, CA 94305

${ }^{7}$ Sean N. Parker Center for Allergy and Asthma Research, Stanford, CA, 94305

${ }^{8}$ Stanford Biobank, Stanford University, Stanford, CA 94305

${ }^{9}$ Chan Zuckerberg Biohub, San Francisco, CA 94158

${ }^{10}$ Howard Hughes Medical Institute, Stanford, CA 94305

\section{Abstract.}

Severe cases of coronavirus disease 2019 (COVID-19), caused by infection with SARS-CoV-2, are characterized by a hyperinflammatory immune response that leads to numerous complications. Production of proinflammatory neutrophil extracellular traps (NETs) has been suggested to be a key factor in inducing a hyperinflammatory signaling cascade, allegedly causing both pulmonary tissue damage and peripheral inflammation. Accordingly, therapeutic blockage of neutrophil activation and NETosis, the cell death pathway accompanying NET 
27 formation, could limit respiratory damage and death from severe COVID-19. Here, we

28 demonstrate that synthetic glycopolymers that activate signaling of the neutrophil checkpoint

29 receptor Siglec-9 suppress NETosis induced by agonists of viral toll-like receptors (TLRs) and

30 plasma from patients with severe COVID-19. Thus, Siglec-9 agonism is a promising therapeutic

31 strategy to curb neutrophilic hyperinflammation in COVID-19.

32

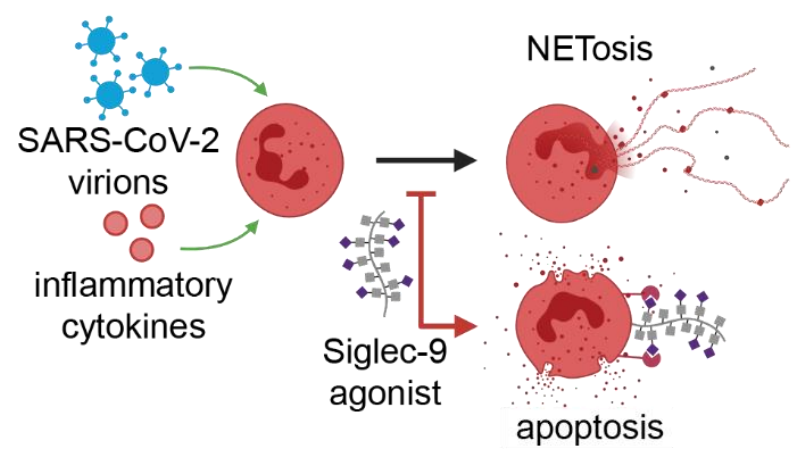

Abstract Figure. In COVID-19, viral pathogen associated molecular patterns and viral-induced

35 cytokines can induce NETosis of neutrophils at the site of infection and in the periphery. Siglec-

369 agonists inhibit COVID-19 plasma-induced NETosis, potentially preventing deleterious

37 hyperinflammatory responses. 


\section{Introduction.}

39 Runaway inflammation in coronavirus disease 2019 (COVID-19) is thought to lead to numerous 40 complications, including potentially fatal pneumonia and acute respiratory distress syndrome 41 (ARDS). ${ }^{1-3}$ While the specific causal factors of inflammation in COVID-19-related ARDS are 42 unknown and likely multifarious, an emerging hypothesis posits that hyperactivation of 43 neutrophils initiates and drives this response (Figure 1). ${ }^{4-12}$ Neutrophils are immune cells of the 44 myeloid lineage that are involved in numerous innate immune functions. It has been suggested 45 that neutrophils drive a hyperinflammatory response in COVID-19 through a death process 46 called NETosis, in which neutrophils rapidly decondense chromatin and spew out a neutrophil 47 extracellular trap (NET), an amalgam of genomic DNA, intracellular proteins (e.g. histones), and 48 tissue-damaging enzymes (e.g. neutrophil elastase, myeloperoxidase). ${ }^{13,14}$ Extracellular DNA 49 and tissue damage from NET-associated enzymes act as proinflammatory signals to other

50 immune cells ${ }^{15-17}$ and are proposed to initiate the hyperinflammatory cascade in COVID-19, 51 leading to ARDS and potentially death. Consistent with this hypothesis, NETs have been 52 extensively observed both at the site of infection (i.e., pulmonary tissue) ${ }^{18-21}$ and in the 53 periphery (i.e., sera and plasma). ${ }^{19,21}$ 


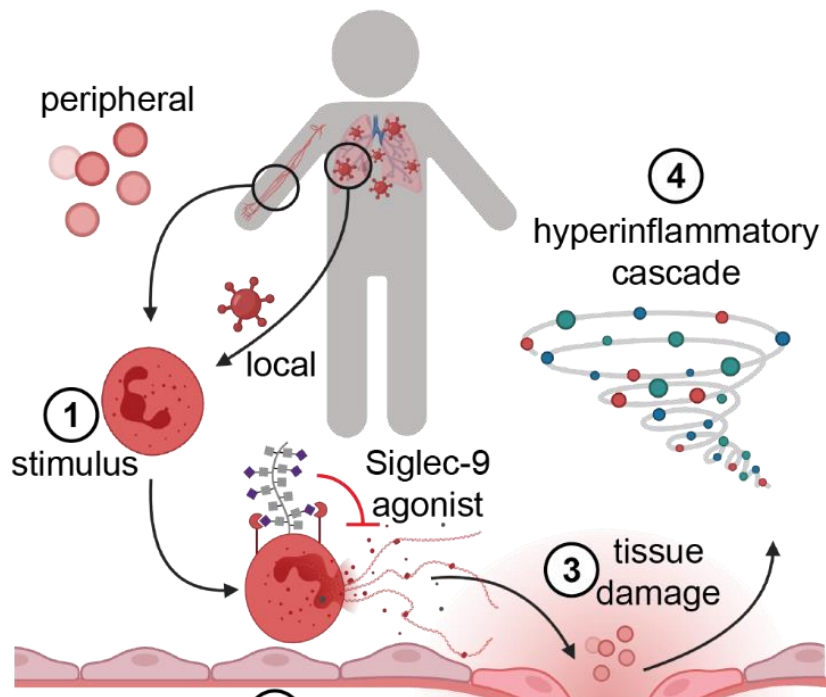

(2) NETosis

Figure 1. Local and peripheral inflammatory stimuli induce NETosis and a subsequent hyperinflammatory cascade in COVID-19. Both local inflammatory stimuli at the site of SARSCoV-2 infection (e.g. virions) and peripheral inflammatory stimuli (e.g. the proinflammatory cytokines IL-8 and G-CSF) associated with COVID-19 have been shown to induce NETosis in vitro. These factors are suspected to be causative agents of NETosis in those tissues, initiating a deleterious hyperinflammatory cascade leading to the symptoms of moderate and severe COVID-19. Agonists of the neutrophil-associated checkpoint receptor Siglec-9 could inhibit NETosis in COVID-19.

Both SARS-CoV-2 virions and serum/plasma from COVID-19 patients have been shown to induce NETosis of neutrophils isolated from healthy donors in vitro, consistent with both the local and peripheral inflammatory responses observed in COVID-19. ${ }^{19,21,22}$ However, the specific signals that induce NETosis in viral disease remain an open question; viral ligands for toll-like receptors (TLRs), host damage-associated molecular patterns, antiviral cytokines (e.g., IL-8 and IFNy), and activated platelets have all been implicated, but which if any of these is sufficient to induce NETosis is still debated. ${ }^{21,23}$ Beyond viral disease, NETosis has been demonstrably 
72 linked to numerous inflammatory pathologies, including thrombosis and sepsis, both of which

73 are observed in patients with COVID-19. ${ }^{4}$ During NETosis, inflammatory stimuli signal

74 neutrophils to import calcium ions, which activates protein arginine deiminase 4 (PADI4). ${ }^{24,25}$

75 PADI4 mediates the conversion of arginine to the deiminated citrulline on histones. ${ }^{25}$ The loss of

76 positive charges induces rapid unwinding of genomic DNA, which eventually ruptures the

77 nucleus and the cell. ${ }^{25}$ When this happens, intracellular contents including genomic DNA, active

78 PADI4, tissue-damaging NET-associated enzymes, and citrullinated histones are emitted into

79 the extracellular space, all of which provoke an inflammatory response. ${ }^{24,25}$ Thus, strategies to

80 curb neutrophil-mediated inflammation could treat both COVID-19 as well as other neutrophilic

81 inflammatory pathologies.

82

83 Transcriptomic analyses of immune cells from severe COVID-19 patients show that neutrophils

84 upregulate the myeloid checkpoint receptor Siglec-9, a member of the sialic acid-binding immunoglobulin-like lectin (Siglec) family that is also found on macrophages and activated T

cells. ${ }^{8,9,26-29}$ This sialoglycan-binding immunosuppressive receptor has an intracellular signaling

87 domain similar to the prominent lymphoid checkpoint molecule PD-1.30,31 And analogous to PD-

88 1, clustering of Siglec-9 by virtue of ligand engagement leads to inhibitory signaling that

89 quenches activation of the immune cells. Both erythrocytes and host-mimicking pathogens

90 have been shown to engage Siglec-9 to suppress neutrophil-mediated immunity. ${ }^{32-35}$

91 Furthermore, engagement of Siglec-9 on primary neutrophils has been shown to induce

92 apoptotic pathways, ${ }^{26}$ in a manner similar to the engagement of Siglec-8 on eosinophils that

93 recently led to an FDA-approved Siglec-8 agonist for eosinophilic inflammatory conditions. ${ }^{36}$

94 Given that Siglec-9 is both anti-inflammatory and pro-apoptotic checkpoint molecule, we

95 hypothesized that engagement of Siglec-9 could simultaneously inhibit proinflammatory NETotic

96 cell death and induce quiet apoptotic cell death in COVID-19-related inflammation. Notably, an

97 agonist (CD24Fc, tradename SACCOVID) of the related myeloid checkpoint receptor Siglec-10 
has recently shown great promise in suppressing viral hyperinflammation and is in a Phase III

99 clinical trial. ${ }^{37,38}$ However, unlike the CD24/Siglec-10, ${ }^{39}$ a specific and high-affinity glycoprotein 100 ligand for Siglec-9 has not been described. ${ }^{40}$

101

102 We recently reported ${ }^{41}$ the design and synthesis of a potent Siglec-9 agonist comprising a lipid103 conjugated glycopolypeptide bearing modified sialic acid residues that Paulson and coworkers 104 had previously found to confer high-affinity and specificity binding to Siglec-9 over other Siglec 105 family members (pS9L, Figure 2). ${ }^{42}$ The lipid group enabled passive insertion into cell 106 membranes, leading to engagement of Siglec-9 in cis on macrophage cell surfaces. This cell107 surface clustering, in turn, induced Siglec-9 signaling suppress macrophage activation. ${ }^{41}$ In this 108 recent study we also designed control glycopolypeptides lacking either Siglec-9 binding glycans 109 (i.e., the lactose-functionalized glycopolypeptide pLac) or a membrane anchoring lipid group 110 (i.e., the soluble glycopolypeptide pS9) (Figure 2). Notably, potent Siglec-9 agonism required 111 membrane anchoring and cis-engagement; the soluble congener pS9 was inable to stimulate

112 Siglec-9 signaling and suppress macrophage activity. We hypothesized that pSL9 might also be 113 able to suppress neutrophil activation and NETosis by clustering Siglec-9 on neutrophils. 


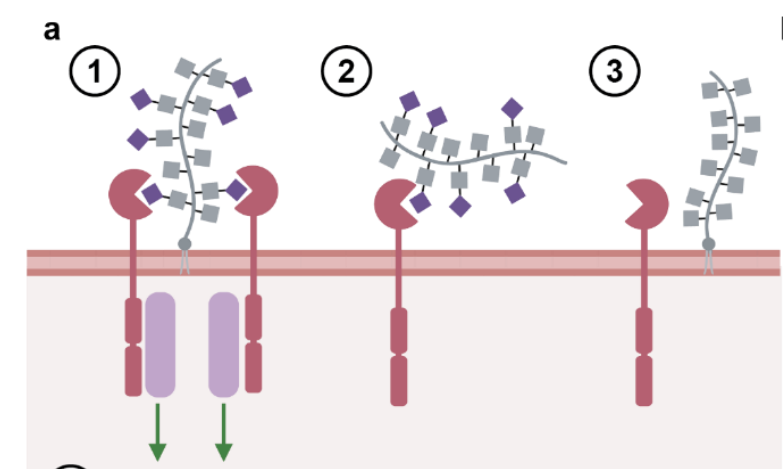

(1) binding; clustering; Siglec-9 signaling

(2) binding; no clustering

(3) no binding

lactose

Siglec-9 ligand
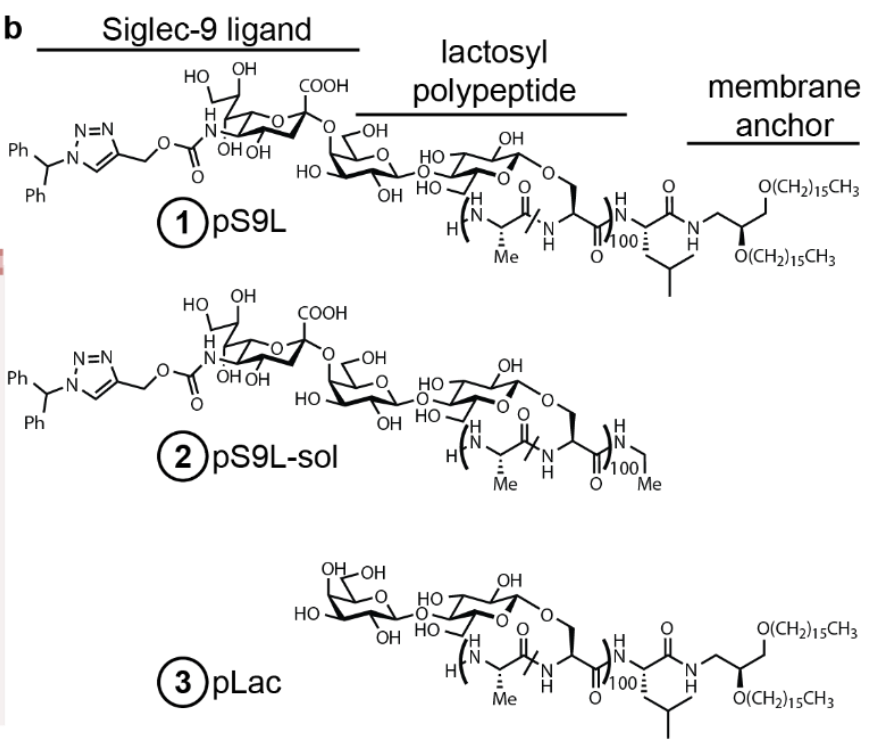

116

117

Figure 2. Synthetic glycopolypeptides bearing high-affinity Siglec-9 ligands cluster and engage Siglec-9 signaling. (a) Membrane-anchored and cis binding glycopolypeptide 1 (pS9L) induces Siglec-9 signaling, while a non-cis binding control polypeptide 2 (pS9L-sol) or a non-binding but membrane-anchored control polypeptide 3 (pLac) do not. (b) Structures of the polypeptides pS9L, pS9L-sol, and pLac. Polypeptides are all based on an O-lactosyl poly-serine-co-alanine scaffold, and in some cases bear terminal Siglec-9 ligands and/or C-terminal membraneanchoring lipids.

Here, we demonstrate that a synthetic cis-binding Siglec-9 agonist (pS9L, Figure $\mathbf{2 b})^{41}$ inhibits NETosis in primary neutrophils in models of local (TLR-7/8 agonist) and peripheral (COVID-19 plasma) COVID-19-associated inflammation. Using time-course live cell microscopy, we showed that TLR-7/8 activation by the nucleoside analog resimiquod ( $R 848$ ) is sufficient to induce specific NETosis in primary human neutrophils. R848 induces rapid citrullination of histone substrates, consistent with PADI4-mediated NETosis, and this process was blocked by Siglec-9 signaling induced by pS9L. SIgnificantly, pS9L inhibited neutrophil NETosis induced by 
132 treatment with plasma from severe COVID-19 patients. In light of these data, we propose that

133 Siglec-9 agonists could be therapeutic agents that inhibit COVID-19-associated inflammation.

\section{Results and Discussion.}

\section{TLR-7/8 agonist R848 induces NETosis of primary neutrophils in vitro.}

137 In COVID-19, evidence of extensive NETosis can be observed in infected lungs, ${ }^{18-21}$ and SARS-

138 CoV-2 virions have been shown to infect and induce NETosis of healthy neutrophils in vitro. ${ }^{20}$

139 These reports implicate TLR-7 and/or TLR-8 in inducing NETosis of neutrophils at the site of 140 infection. ${ }^{20,43}$ Notably, TLR-7 and TLR-8 are ssRNA receptors with numerous substrates 141 identified in the SARS-CoV-2 genome. ${ }^{44}$ Furthermore, consistent with the hypothesis that 142 SARS-CoV-2 induces TLR-7/8-mediated immunity, TLR-7 deficiency is associated with severe 143 COVID-19. ${ }^{45}$ Thus, agonists of TLR-7/8 may provide a convenient means of modeling local inflammation induced by viral infection in vitro without using live virus.

We assayed TLR agonists using the live-cell imaging techniques described by Gupta and coworkers. ${ }^{46}$ In this assay, freshly isolated neutrophils are cultured in low-serum media in the presence of a fluorogenic and membrane impermeable DNA-intercalating dye (Cytotox Green). Upon genomic DNA-externalization by NETosis, dye intercalates and fluorescence increases. As previously demonstrated, ${ }^{46}$ because NETs are much larger than the nuclei of apoptotic cells,

151 NETotic cells yield much larger areas of fluorescence than apoptotic cells, as observed by 152 microscopy. Thus, apoptotic cells can be filtered out by only counting large (i.e., >>100 $\mu \mathrm{m}^{2}$ ) 153 fluorescent objects.

155 We found that a TLR-7/8 agonist, R848, was sufficient to induce NETosis of healthy neutrophils 156 in vitro (Figure 3a-c, Figure S1). We also assayed the citrullination status of the PADI4 157 substrate $\mathrm{H} 3$ by Western blot, and observed that $\mathrm{R} 848$ rapidly induced citrullination at $\mathrm{R} 2, \mathrm{R} 8$, 
and R17 (Figure S2). Additionally, we performed quantitative phosphoproteomics ${ }^{47}$ with lysates of neutrophils treated with media, phorbol-12-myristate-13-acetate (PMA), or R848 (Figure S3, Table S1). We observed similar results to previously published datasets using neutrophils stimulated with either $\mathrm{R} 848^{48}$ or $\mathrm{PMA}^{49}$. Furthermore, several phosphosites were found to be differentially regulated in both datasets, including those involved in neutrophil degranulation and calcium flux, consistent with the described mechanism of NETotic death. ${ }^{24,49}$ These results indicate that the TLR-7/8 agonist R848 induces NETosis in primary neutrophils. Thus, this can be used to model local inflammation associated with viral infection, including in COVID-19.

\section{A Siglec-9 agonist inhibits TLR-7/8-induced NETosis via SHP-1.}

Previous studies by von Guten and coworkers have shown that engagement of Siglec-9 leads to apoptotic and nonapoptotic death pathways as well as immunosuppression in neutrophils. ${ }^{26,32}$ Thus, we hypothesized that Siglec-9 mediated immunosuppression and cell death could override the NETotic effect of antiviral TLR signaling. To assay this, we employed the synthetic Siglec-9 cis-binding agonist, pS9L, that we have previously described. ${ }^{41}$ pS9L is a lipid-tethered glycopolypeptide, biomimetically inspired by native mucin proteins (Figure 2). When the lipid inserts into the cell membrane, the polypeptide backbone can adopt extended conformations, ${ }^{50}$ resulting in presentation of multivalent high-affinity Siglec-9 cis ligands. ${ }^{41}$ We have previously observed that presentation in this manner results in binding and clustering with Siglec-9 in cis. ${ }^{41}$ We additionally used two control glycopolypeptides: pLac, a lipid-tethered glycopolypeptide based on the same scaffold as PS9L, but lacking terminal Siglec-9 binding sialosides, and pS9L-sol, a soluble lipid-free analogue of pS9L incapable of binding in cis (Figure 2, S4).

We assayed anti-NETotic activity by cotreatment of glycopolypeptide (500 nM) with R848 (10 $\mu \mathrm{M}$ ) in primary neutrophils in the live-cell assay described above (Figure 3). We observed that pS9L was sufficient to inhibit NETosis induced by R848 treatment (Figure 3a-c). Moreover, 
184 neither control polymer inhibited R848-induced NETosis (Figure 3d). We also confirmed that 185 pS9L inhibits NETosis comparably to high concentrations of crosslinked anti-Siglec-9 antibody 186 (clone 191240) (Figure S5). ${ }^{51,52}$ Additionally, we found that treatment with pS9L induced an 187 oxidative burst in primary neutrophils (Figure S6), which von Guten and coworkers described as 188 an important signaling step of Siglec-9-induced apoptotic signaling. ${ }^{26}$ Furthermore, the oxidative 189 burst was inhibited by the addition of the SHP-1/2 inhibitor NSC-87877, suggesting that SHP-1 190 and/or SHP-2 mediate pS9L-induced oxidative burst in neutrophils, consistent with Siglec-9 191 engagement (Figure S6b).

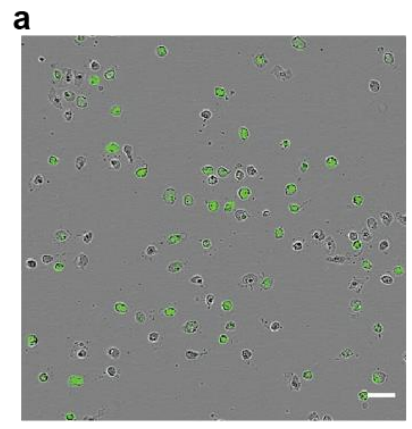

Untreated

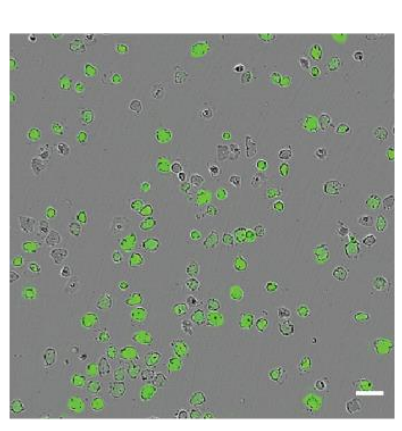

R848

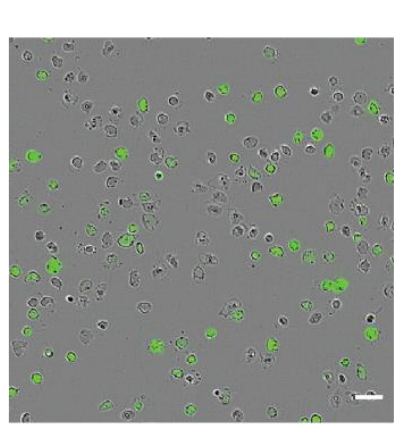

$\mathrm{R} 848+\mathrm{pS9L}$
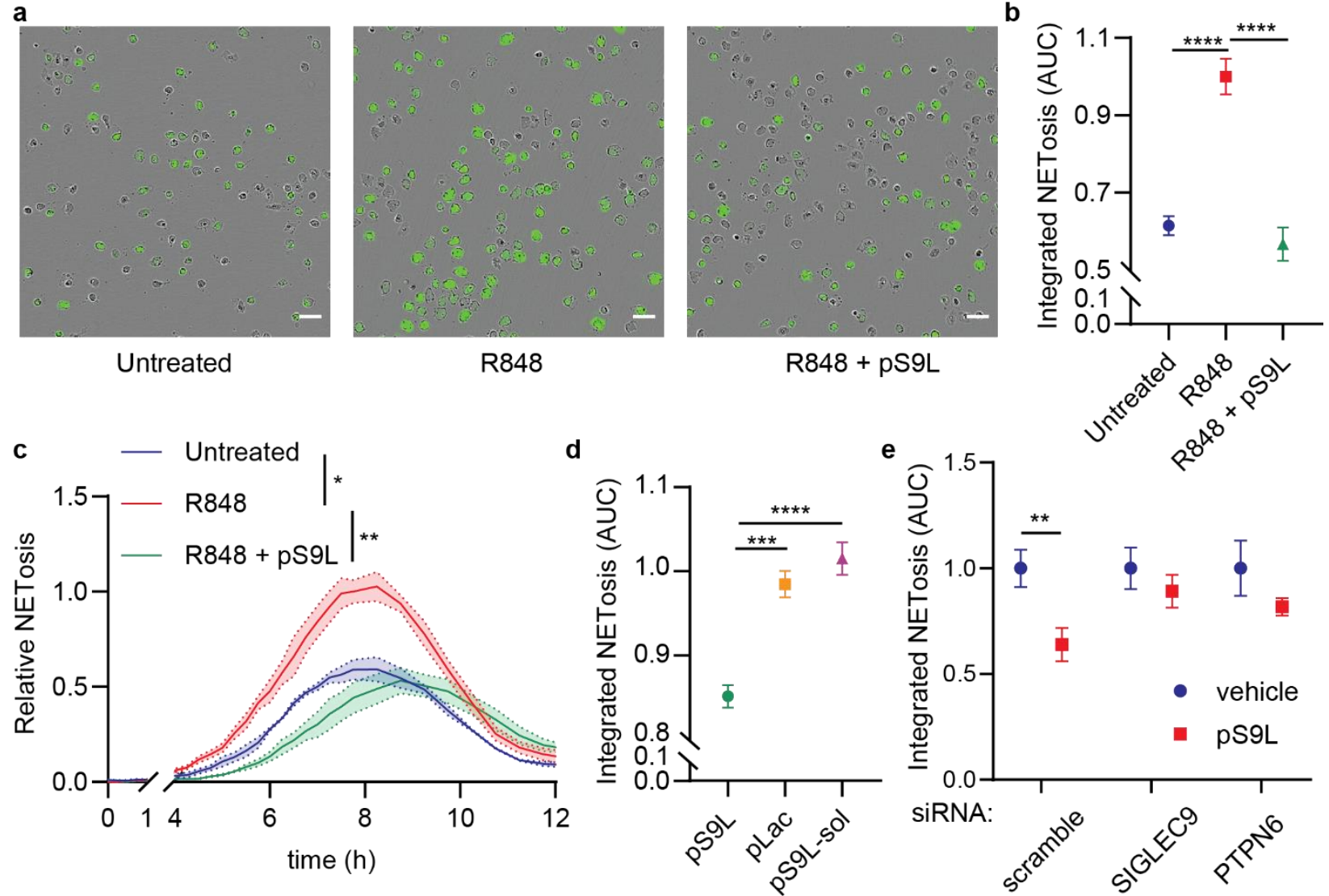

193 Figure 3. A cis-binding Siglec-9 agonist (pS9L) inhibits R848-induced NETosis via Siglec-9 and 194 SHP-1. (a-c) Primary neutrophils were cotreated with R848 (10 $\mu \mathrm{M})$ and glycopolypeptide (500 $195 \mathrm{nM}$ ) in IMDM supplemented 0.5\% hiFBS containing the membrane impermeable DNA 196 intercalators Cytotox Green or Red (250 nM). Images were acquired by fluorescence 
microscopy every $15 \mathrm{~min}$ for $12 \mathrm{~h}$. The area of all green fluorescent objects $>300 \mu \mathrm{m}^{2}$ was quantified and the total area was averaged across three images per well. Relative NETosis was determined by normalizing to the maximal NET area from R848 treatment alone ( $t=8 \mathrm{~h})$. (a) Representative phase contrast and fluorescence images from $t=8 \mathrm{~h}$. Scale bars indicate 40 $\mu \mathrm{m}$. (b) Quantitation of NETosis over time as area under the curve in (c). Error bars represent SD. (c) NET formation and degradation as a function of time. Error bands represent SEM. (d) Treatment of R848-stimulated neutrophils with various glycopolypeptides. Error bars represent SD. (e) pS9L is a mucin-like glycopolypeptide that bears high affinity and specific ligands for Siglec-9 and is functionalized with a membrane-tethering lipid tail. (f) HL-60 cells were transfected with siRNAs against SIGLEC9 (encoding Siglec-9), PTPN6 (encoding SHP-1), or a scrambled control and then grown for two days. Cells were then cotreated with R848 (10 $\mu \mathrm{M})$ and vehicle or pS9L (500 nM). Relative NETosis is determined as in (b), except all objects $>200$ $\mu \mathrm{m}^{2}$ were quantified and the R848 maximum in $\mathrm{dHL}-60$ 's was observed at $2.5 \mathrm{~h}$ post induction. Error bars represent SD. Statistics were determined by two-way ANOVA (b) or one-way ANOVA $(\mathbf{c}, \mathbf{d}, \mathbf{f}) .{ }^{*} p<0.05 ;{ }^{* *} p<0.01 ;{ }^{* * *} p<0.001 ;{ }^{* \star * *} p<0.0001$.

We performed quantitative phosphoproteomics using lysates of R848-stimulated primary neutrophils cotreated with vehicle, pS9L, or pLac (Figure S3, Table S2). Notably, we found increased phosphorylation of hyccin (HYCCl / FAM126A), a key component in phosphorylation of phosphoinositides,$^{53}$ a class of signaling molecules implicated in mediating NETosis. ${ }^{54}$ Additionally, we observed increased phosphorylation of RASAL3 (RASL3), a negative regulator of the MAPK signaling pathway. ${ }^{55}$ These data suggest that $\mathrm{pS9L}$ inhibits the calcium flux and NADPH activity necessary for NETosis, as well as the MAPK-suppressive effects that have been previously described for PS9L in macrophages. ${ }^{41}$ 
222 To determine whether the anti-NETotic effect of pS9L is specifically mediated by Siglec-9

223 signaling, we recapitulated our results in the HL-60 cell line. The HL-60 line is a promyelocytic

224 leukemia that can be differentiated into a neutrophil-like cells (dHL-60) using all-trans retinoic

225 acid (ATRA, $100 \mathrm{nM}$ ) and dimethylsulfoxide (DMSO, 1.25\% v/v), which have previously been

226 used to study NETosis in vitro. ${ }^{31,56}$ We observed that R848 induced NETosis in dHL-60 cell's

227 (Figure S7). Furthermore, we observed that pS9L inhibited NETosis and that siRNA knockdown

228 of Siglec-9 (encoded by SIGLEC9) or SHP-1 (encoded by PTPN6) abrogated the effect of pS9L

229 (Figure 3e, S8, S9). Therefore, the Siglec-9 agonist pS9L inhibits TLR7/8-induced NETosis via

230 Siglec-9 and SHP-1. This suggests that Siglec-9 agonists could inhibit NETosis at the site of

231 viral infection, thus preventing pulmonary inflammation in COVID-19.

232

233

Siglec-9 is upregulated in severe COVID-19 and can inhibit NETosis induced by COVID-19

234 plasma.

235 Sera and plasma from COVID-19 patients have been shown to be sufficient to induce NETosis

236 of neutrophils isolated from healthy donors in vitro. ${ }^{19,21}$ The causes of this are unclear, however

237 potential factors include viral TLR ligands, damage-associated molecular patterns that bind

238 TLRs, activated platelets, and (pro)inflammatory cytokines. Recent reports have described

239 increased levels of neutrophil-activating cytokines, predominantly IL-8 and G-CSF. ${ }^{7}$ Consistent

240 with this observation, we also observed that the combination of IL-8 and G-CSF was sufficient to

241 induce NETosis in vitro (Figure S10). Additionally, transcriptomic analyses of peripheral myeloid

242 cells $^{8}$ and neutrophils ${ }^{9}$ in COVID-19 patients have observed increased SIGLEC9 expression

243 (Figure 4a, S11) and PADI4 expression (Figure 4b, S11). We hypothesize that this is an

244 exhaustion-like phenotype of induced Siglec-9 expression of hyper-NETotic neutrophils, similar

245 to what has been observed with Siglec-9 on exhausted tumor-infiltrating T cells. ${ }^{51}$ This makes

246 Siglec-9 an attractive target for therapeutic blockade of hyperinflammatory NETosis in COVID-

24719. 

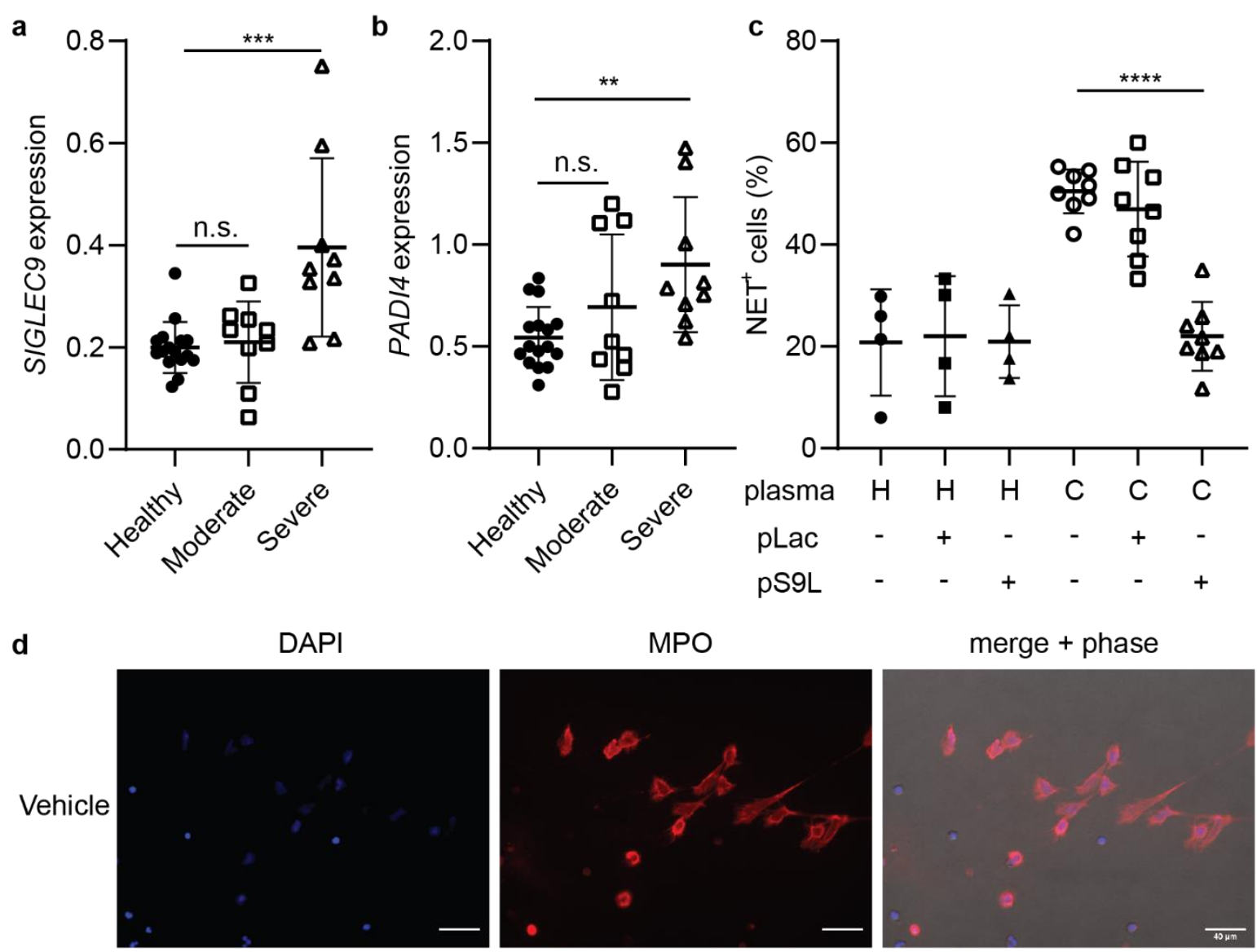

MPO

merge + phase
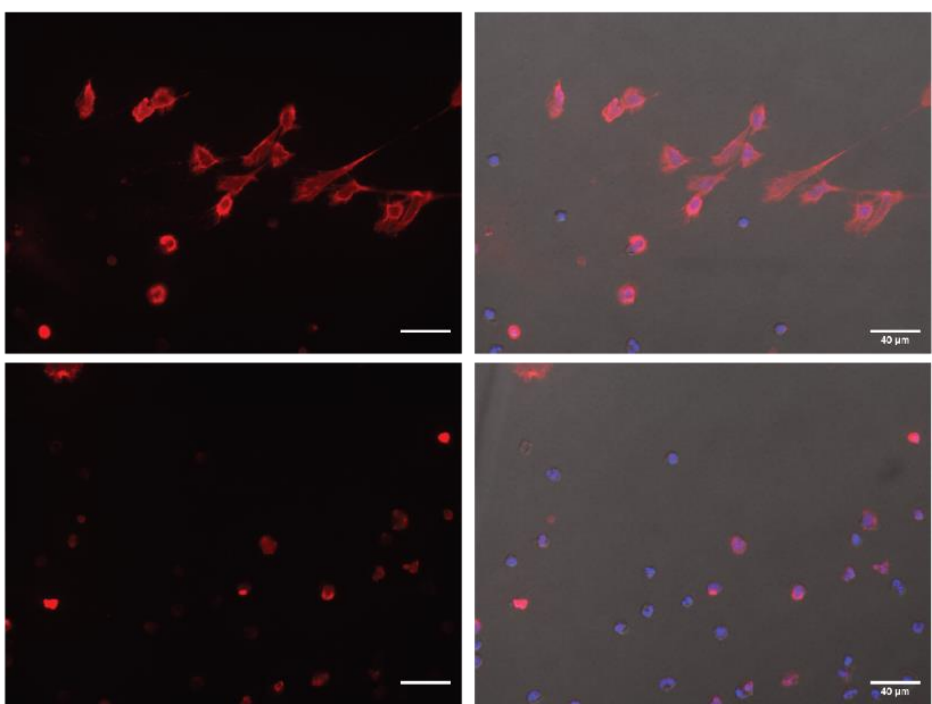

Figure 4. A Siglec-9 agonist inhibits NETosis of neutrophils induced by COVID-19 plasma. (a,b) Analysis of publicly available single-cell transcriptomics data ${ }^{8}$ for SIGLEC9 expression (a) and PADI4 expression (b) on neutrophils in peripheral blood from healthy donors or COVID-19 patients. Error bars represent SD. Statistics were determined using mixed effects model. ${ }^{* *}=p$ $<0.01 ;{ }^{* *}=\mathrm{p}<0.001(\mathbf{c}, \mathbf{d})$ Primary neutrophils were cultured in undiluted and citrate anticoagulated plasma from healthy donors or COVID-19 patients for $4 \mathrm{~h}$. Cells were fixed, stained for extracellular myeloperoxidase, and imaged in DAPI imaging media by fluorescence microscopy. Cells were treated in technical triplicate and imaged across multiple fields of view. 
(c) Proportion of NET-positive cells (\%) across all fields of view. Each dot represents and

258 individual plasma sample. (d) Representative images from a COVID-19 patient plasma sample 259 with or without pS9L. Error bars represent SD. Statistics were determined using mixed effects 260 models to account for samples using repeat neutrophil donors. ${ }^{* \star * *}=p<0.0001$.

To test the hypothesis that pS9L can inhibit NETosis induced by COVID-19 plasma, we treated neutrophils isolated from whole blood of healthy donors with citrate-anticoagulated heterologous plasma from healthy donors or COVID-19 patients. Neutrophils in undiluted plasma were cotreated with pS9L (500 nM), the non-binding analog pLac (500 nM), or vehicle. To satisfy biosafety restrictions, cells were incubated in the presence of COVID-19 plasma for $4 \mathrm{~h}$ and then fixed before assaying for extracellular complexes of myeloperoxidase (MPO) and DNA (DAPI) (Figure 4c,d). The combination of these stains, which when observed extracellularly is indicative of NETosis, has been previously used to identified $\mathrm{NET}^{+}$cells in the context of COVID-19. ${ }^{20}$ We observed that COVID-19 plasma induced NETosis of neutrophils from healthy 271 donors, and that this effect was inhibited by pS9L (Figure 4c,d). This is indicated by the distinct 272 web-like morphology of NETs (Figure 4d). Furthermore, we observe that pLac does not inhibit

273 COVID-19-induced NETosis, and that neither polymer effects basal NETosis of in vitro cultured 274 neutrophils (Figure 4c). Additionally, we performed similar experiments staining neutrophils 275 treated with 10\% plasma in IMDM (Figure S12) or undiluted plasma (Figure S13) for 276 extracellular H1/DNA complexes, another marker of NETs, ${ }^{58-60}$ and observed comparable 277 results.

279 Collectively, these data demonstrate that Siglec-9 agonists are sufficient to inhibit NETosis 280 induced by COVID-19 patient plasma, and thus could inhibit peripheral inflammation in patients 281 with COVID-19. Additionally, Siglec-9 agonists could resolve NET-associated pathologies 282 observed in COVID-19 and elsewhere such as immunothrombosis ${ }^{21}$ and sepsis..$^{4,5}$ 


\section{Conclusion.}

284 Here, we have demonstrated that Siglec-9 agonists can inhibit NETosis induced by COVID-19285 associated proinflammatory signals. Thus, if NETosis is a causal factor of COVID-19-related 286 hyperinflammation as has been hypothesized, Siglec-9 is a therapeutic target to inhibit 287 potentially fatal hyperinflammation associated with COVID-19 in an analogous fashion to the 288 highly effective therapeutics currently aimed at the Siglec-10/CD24 interaction. A CD24-Fc 289 fusion protein has been shown to both engage Siglec-10 as an immune checkpoint on 290 macrophages and sequester the nuclear protein HMGB1, which can act as a damage 291 associated molecular pattern by engaging TLR4. ${ }^{39}$ The Siglec-9 agonists described here have 292 previously been shown to inhibit macrophage TLR4 signaling and engage macrophage Siglec9. ${ }^{41}$ Thus, Siglec-9 agonists may be multipurpose therapeutics, able to both inhibit the clinically unaddressed problem of proinflammatory NETosis and also subsequent inflammatory signaling from tissue damage that is currently being clinically investigated. Finally, Siglec- 9 agonists have the potential to expand beyond ARDS to other NET-related pathologies such as thrombosis, ${ }^{61,62}$

297 atherosclerosis, ${ }^{63}$ and cystic fibrosis. ${ }^{64}$

\section{Acknowledgements.}

300 The authors would like to thank the following individuals at the Stanford COVID-19 Biobank for 301 enabling this work: Nancy Zhao, Rosemary Vergara, Julia McKechnie, Aaron Wilk, Lauren de la 302 Parte, Kathleen Whittle Dantzler, Maureen Ty, Nimish Kathale, Arjun Rustagi, Giovanny 303 Martinez-Colon, Geoff Ivison, Ruoxi Pi, Maddie Lee, Rachel Brewer, Taylor Hollis, Andrea 304 Baird, Michele Ugur, Drina Bogusch, Georgie Nahass, Kazim Haider, Thanmayi Ranganath, 305 Kim Quyen Thi Tran, Laura Simpson, Michal Tal, Iris Chang, Evan Do, Andrea Fernandes, Shu306 Chen Lyu, Wenming Zhang, Monali Manohar, James Krempski, Jonasel Roque, Hena Naz 307 DinRosen Mann, Anita Visweswaran, Elizabeth J. Zudock, Kathryn Jee, Komal Kumar, Jennifer 308 A. Newberry, James V. Quinn, Donald Schreiber, and Andra L. Blomkalns. The authors would 
additionally like to thank: Rishi Kulkarni and Susan Holmes for their advice on statistics; Gabby

310 Tender for her assistance with fluorescence microscopy. This work was supported by a grant

311 from the National Institutes of Health to C.R.B. (CA227942). C.S.D was supported by a National

312 Science Foundation Graduate Research Fellowship (DGE-114747) and a ChEM-H affiliated

313 Stanford Interdisciplinary Gradate Fellowship. N.M.R. was funded through an NIH Predoctoral to

314 Postdoctoral Transition Award (Grant K00 CA212454-05). J.C.S. was supported by an NIH/NCI

315 F32 postdoctoral fellowship (Grant 1F32CA250324-01). A.J.W. was supported by the Stanford

316 Medical Scientist Training Program (T32 GM007365-44) and the Stanford Bio-X Interdisciplinary

317 Graduate Fellowship. C.A.B. was supported by the Burroughs Wellcome Fund Investigators in

318 the Pathogenesis of Infectious Diseases \#1016687 and the Bill and Melinda Gates Foundation

319 (OPP1113682). C.A.B. is the Tashia and John Morgridge Faculty Scholar in Pediatric

320 Translational Medicine from the Stanford Maternal Child Healthy Research Institute and an

321 Investigator of the Chan Zuckerberg Biohub. Figure illustrations were created using

322 BioRender.com.

\section{Conflicts of Interest.}

325 C.S.D. and C.R.B. are coinventors on a patent application for cis-binding Siglec agonist 326 glycopolymers as immune suppressants (USPTO63046140). C.R.B. is a co-founder and 327 Scientific Advisory Board member of Lycia Therapeutics, Palleon Pharmaceuticals, Enable 328 Bioscience, Redwood Biosciences (a subsidiary of Catalent), and InterVenn Bio, and a member 329 of the Board of Directors of Eli Lily \& Company. C.A.B. is a Scientific Advisory Board member of 330 Catamaran Bio. 


\section{References.}

(1) Cao, X. COVID-19: Immunopathology and Its Implications for Therapy. Nat. Rev. Immunol. 2020, 2019, 2019-2020. https://doi.org/10.1038/s41577-020-0308-3.

(2) Mason, R. J. Pathogenesis of COVID-19 from a Cell Biology Perspective. Eur. Respir. J. 2020, 55 (4), 9-11. https://doi.org/10.1183/13993003.00607-2020.

(3) Tay, M. Z.; Poh, C. M.; Rénia, L.; MacAry, P. A.; Ng, L. F. P. The Trinity of COVID-19: Immunity, Inflammation and Intervention. Nat. Rev. Immunol. 2020, 20 (6), 363-374. https://doi.org/10.1038/s41577-020-0311-8.

(4) Thierry, A. R.; Roch, B. NETs By-Products and Extracellular DNA May Play a Key Role in COVID-19 Pathogenesis : Incidence on Patient Monitoring and Therapy. 2020, No. April, 1-21. https://doi.org/10.20944/preprints202004.0238.v1.

(5) Barnes, B. J.; Adrover, J. M.; Baxter-Stoltzfus, A.; Borczuk, A.; Cools-Lartigue, J.;

Crawford, J. M.; Daßler-Plenker, J.; Guerci, P.; Huynh, C.; Knight, J. S.; et al. Targeting Potential Drivers of COVID-19: Neutrophil Extracellular Traps. J. Exp. Med. 2020, 217 (6), 1-7. https://doi.org/10.1084/jem.20200652.

(6) Liu, J.; Liu, Y.; Xiang, P.; Pu, L.; Xiong, H.; Li, C.; Zhang, M.; Tan, J.; Xu, Y.; Song, R.; et al. Neutrophil-to-Lymphocyte Ratio Predicts Severe Illness Patients with 2019 Novel Coronavirus in the Early Stage. medRxiv 2020, 807, 2020.02.10.20021584. https://doi.org/10.1101/2020.02.10.20021584.

(7) Bendib, I.; De Chaisemartin, L.; Granger, V.; Schlemmer, F.; Maitre, B.; Hüe, S.; Surenaud, M.; Beldi-Ferchiou, A.; Carteaux, G.; Razazi, K.; et al. Neutrophil Extracellular Traps Are Elevated in Patients with Pneumonia-Related Acute Respiratory Distress Syndrome. Anesthesiology 2019, 130 (4), 581-591. https://doi.org/10.1097/ALN.0000000000002619. 
(8) Schulte-Schrepping, J.; Reusch, N.; Paclik, D.; Baßler, K.; Schlickeiser, S.; Zhang, B.; Krämer, B.; Krammer, T.; Brumhard, S.; Bonaguro, L.; et al. Severe COVID-19 Is Marked by a Dysregulated Myeloid Cell Compartment. Cell 2020, 1-22. https://doi.org/10.1016/j.cell.2020.08.001.

(9) Aschenbrenner, A. C.; Mouktaroudi, M.; Krämer, B.; Antonakos, N. Neutrophil Transcriptomes Stratify COVID-19 Patients Signatures in Blood. medRxiv 2020, 1-56.

(10) Wilk, A. J.; Rustagi, A.; Zhao, N. Q.; Roque, J.; Martínez-Colón, G. J.; McKechnie, J. L.; Ivison, G. T.; Ranganath, T.; Vergara, R.; Hollis, T.; et al. A Single-Cell Atlas of the Peripheral Immune Response in Patients with Severe COVID-19. Nat. Med. 2020, 26 (7), 1070-1076. https://doi.org/10.1038/s41591-020-0944-y.

(11) Bost, P.; De Sanctis, F.; Canè, S.; Ugel, S.; Donadello, K.; Castellucci, M.-N.; Eyal, D.; Fiore, A.; Anselmi, C.; Barouni, R. M.; et al. Deciphering the State of Immune Silence in Fatal COVID-19 Patients. medRxiv 2020, 2020.08.10.20170894.

(12) Overmyer, K. A.; Shishkova, E.; Miller, I. J.; Balnis, J.; Bernstein, M. N.; Peters-Clarke, T. M.; Meyer, J. G.; Quan, Q.; Muehlbauer, L. K.; Trujillo, E. A.; et al. Large-Scale MultiOmic Analysis of COVID-19 Severity. Cell Syst. 2020, 1-18. https://doi.org/10.1016/j.cels.2020.10.003.

(13) Remijsen, Q.; Kuijpers, T. W.; Wirawan, E.; Lippens, S.; Vandenabeele, P.; Vanden Berghe, T. Dying for a Cause: NETosis, Mechanisms behind an Antimicrobial Cell Death Modality. Cell Death Differ. 2011, 18 (4), 581-588. https://doi.org/10.1038/cdd.2011.1.

(14) Brinkmann, V.; Reichard, U.; Goosmann, C.; Fauler, B.; Uhlemann, Y.; Weiss, D. S.; Weinrauch, Y.; Zychlinsky, A. Neutrophil Extracellular Traps Kill Bacteria. Science (80-. ). 2004, 303 (5663), 1532-1535. https://doi.org/10.1126/science.1092385. 
(15) Daniel, C.; Leppkes, M.; Muñoz, L. E.; Schley, G.; Schett, G.; Herrmann, M. Extracellular DNA Traps in Inflammation, Injury and Healing. Nat. Rev. Nephrol. 2019, 15 (9), 559575. https://doi.org/10.1038/s41581-019-0163-2.

(16) Xu, J.; Zhang, X.; Monestier, M.; Esmon, N. L.; Esmon, C. T. Extracellular Histones Are Mediators of Death through TLR2 and TLR4 in Mouse Fatal Liver Injury. J. Immunol. 2011, 187 (5), 2626-2631. https://doi.org/10.4049/jimmunol.1003930.

(17) Lood, C.; Blanco, L. P.; Purmalek, M. M.; Carmona-Rivera, C.; De Ravin, S. S.; Smith, C. K.; Malech, H. L.; Ledbetter, J. A.; Elkon, K. B.; Kaplan, M. J. Neutrophil Extracellular Traps Enriched in Oxidized Mitochondrial DNA Are Interferogenic and Contribute to Lupus-like Disease. Nat. Med. 2016, 22 (2), 146-153. https://doi.org/10.1038/nm.4027.

(18) Radermecker, C.; Detrembleur, N.; Guiot, J.; Cavalier, E.; Henket, M.; Cataldo, D.; Delvenne, P.; Marichal, T.; Chemistry, M.; Biology, D. Neutrophil Extracellular Traps Infiltrate the Lung Vascular, Interstitial and Airway Compartments in Severe Covid-19. J. Leukoc. Biol. 2020, 217 (12), 1-33.

(19) Zuo, Y.; Yalavarthi, S.; Shi, H.; Gockman, K.; Zuo, M.; Madison, J. A.; Blair, C.; Weber, A.; Barnes, B. J.; Egeblad, M.; et al. Neutrophil Extracellular Traps in COVID19. J. Clin. Invest. 2020, 5 (11). https://doi.org/10.3390/cells9061494.

(20) Veras, F. P.; Pontelli, M.; Silva, C.; Toller-Kawahisa, J.; de Lima, M.; Nascimento, D.; Schneider, A.; Caetite, D.; Rosales, R.; Colon, D.; et al. SARS-CoV-2 Triggered Neutrophil Extracellular Traps (NETs) Mediate COVID-19 Pathology. J. Exp. Med. 2020, 217 (12). https://doi.org/10.1101/2020.06.08.20125823.

(21) Middleton, E. A.; He, X.-Y.; Denorme, F.; Campbell, R. A.; Ng, D.; Salvatore, S. P.; Mostyka, M.; Baxter-Stoltzfus, A.; Borczuk, A. C.; Loda, M.; et al. Neutrophil Extracellular Traps (NETs) Contribute to Immunothrombosis in COVID-19 Acute Respiratory Distress 
Syndrome. Blood 2020, 136 (10). https://doi.org/10.1182/blood.2020007008.

404

405

406

407

408

409

410

411

412

413

414

415

416

417

418

419

420

421

422

423

424

425

(22) Veras, F.; et al. SARS-CoV-2 Triggered Neutrophil Extracellular Traps (NETs) Mediate COVID-19 Pathology. MedRxiv 2020.

(23) Schönrich, G.; Raftery, M. J. Neutrophil Extracellular Traps Go Viral. Front. Immunol. 2016, 7 (SEP), 11-14. https://doi.org/10.3389/fimmu.2016.00366.

(24) Thiam, H. R.; Wong, S. L. Cellular Mechanisms of NETosis. Annu. Rev. Cell Dev. Biol. 2020, $8(41), 1-28$.

(25) Leshner, M.; Wang, S.; Lewis, C.; Zheng, H.; Chen, X. A.; Santy, L.; Wang, Y. PAD4 Mediated Histone Hypercitrullination Induces Heterochromatin Decondensation and Chromatin Unfolding to Form Neutrophil Extracellular Trap-like Structures. Front. Immunol. 2012, 3 (October), 1-11. https://doi.org/10.3389/fimmu.2012.00307.

(26) Von Gunten, S.; Yousefi, S.; Seitz, M.; Jakob, S. M.; Schaffner, T.; Seger, R.; Takala, J.; Villiger, P. M.; Simon, H. U. Siglec-9 Transduces Apoptotic and Nonapoptotic Death Signals into Neutrophils Depending on the Proinflammatory Cytokine Environment. Blood 2005, 106 (4), 1423-1431. https://doi.org/10.1182/blood-2004-10-4112.

(27) Angata, T.; Varki, A. Cloning, Characterization, and Phylogenetic Analysis of Siglec-9, a New Member of the CD33-Related Group of Siglecs: Evidence for Co-Evolution with Sialic Acid Synthesis Pathways. J. Biol. Chem. 2000, 275 (29), 22127-22135. https://doi.org/10.1074/jbc.M002775200.

(28) Lizcano, A.; Secundino, I.; Dohrmann, S.; Corriden, R.; Rohena, C.; Diaz, S.; Ghosh, P.; Deng, L.; Nizet, V.; Varki, A. Erythrocyte Sialoglycoproteins Engage Siglec-9 on Neutrophils to Suppress Activation. Blood 2017, 129 (23), 3100-3110. https://doi.org/10.1182/blood-2016-11-751636. 
(29) Adams, O. J.; Stanczak, M. A.; von Gunten, S.; Läubli, H. Targeting Sialic Acid-Siglec Interactions to Reverse Immune Suppression in Cancer. Glycobiology 2017, No. January 2018, 1-8. https://doi.org/10.1093/glycob/cwx108.

(30) Duan, S.; Paulson, J. C. Siglecs as Immune Cell Checkpoints in Disease. Annu. Rev. Immunol. 2020, 38 (1), 365-395. https://doi.org/10.1146/annurev-immunol-102419035900.

(31) Zhang, J. Q.; Nicoll, G.; Jones, C.; Crocker, P. R. Siglec-9, a Novel Sialic Acid Binding Member of the Immunoglobulin Superfamily Expressed Broadly on Human Blood Leukocytes. J. Biol. Chem. 2000, 275 (29), 22121-22126. https://doi.org/10.1074/jbc.M002788200.

(32) Lizcano, A.; Secundino, I.; Dohrmann, S.; Corriden, R.; Rohena, C.; Diaz, S.; Ghosh, P.; Deng, L.; Nizet, V.; Varki, A. Erythrocyte Sialoglycoproteins Engage Siglec-9 on Neutrophils to Suppress Activation. Blood 2017, 129 (23), 3100-3110. https://doi.org/10.1182/blood-2016-11-751636.

(33) Bornhöfft, K. F.; Galuska, S. P. Glycans as Modulators for the Formation and Functional Properties of Neutrophil Extracellular Traps : Used by the Forces of Good and Evil. Front. Immunol. 2019, 10 (May), 1-9. https://doi.org/10.3389/fimmu.2019.00959.

(34) Carlin, A. F.; Uchiyama, S.; Chang, Y. C.; Lewis, A. L.; Nizet, V.; Varki, A. Molecular Mimicry of Host Sialylated Glycans Allows a Bacterial Pathogen to Engage Neutrophil Siglec-9 and Dampen the Innate Immune Response. Blood 2009, 113 (14), 3333-3336. https://doi.org/10.1182/blood-2008-11-187302.

(35) Khatua, B.; Bhattacharya, K.; Mandal, C. Sialoglycoproteins Adsorbed by Pseudomonas Aeruginosa Facilitate Their Survival by Impeding Neutrophil Extracellular Trap through Siglec-9. J. Leukoc. Biol. 2012, 91 (April), 641-655. https://doi.org/10.1189/jlb.0511260. 
450

451

452

453

454

455

456

457

458

459

460

461

462

463

464

465

466

467

468

469

470

471

472

(36) Youngblood, B. A.; Brock, E. C.; Leung, J.; Falahati, R.; Bochner, B. S.; Rasmussen, H. S.; Peterson, K.; Bebbington, C.; Tomasevic, N. Siglec-8 Antibody Reduces Eosinophils and Mast Cells in a Transgenic Mouse Model of Eosinophilic Gastroenteritis. JCl Insight 2019, 4 (19). https://doi.org/10.1172/jci.insight.126219.

(37) Tian, R. R.; Zhang, M. X.; Liu, M.; Fang, X.; Li, D.; Zhang, L.; Zheng, P.; Zheng, Y. T.; Liu, Y. CD24Fc Protects against Viral Pneumonia in Simian Immunodeficiency VirusInfected Chinese Rhesus Monkeys. Cell. Mol. Immunol. 2020, 17 (8), 887-888. https://doi.org/10.1038/s41423-020-0452-5.

(38) Tian, R. R.; Zhang, M. X.; Zhang, L. T.; Zhang, P.; Ma, J. P.; Liu, M.; Devenport, M.; Zheng, P.; Zhang, X. L.; Lian, X. D.; et al. CD24 and Fc Fusion Protein Protects SIVmac239-Infected Chinese Rhesus Macaque against Progression to AIDS. Antiviral Res. 2018, 157 (June), 9-17. https://doi.org/10.1016/j.antiviral.2018.07.004.

(39) Chen, G. Y.; Tang, J.; Zheng, P.; Liu, Y. CD24 and Siglec-10 Selectively Repress Tissue Damage - Induced Immune Responses. Science (80-. ). 2009, 323 (5922), 1722-1725. https://doi.org/10.1126/science.1168988.

(40) Läubli, H.; Varki, A. Sialic Acid - Binding Immunoglobulin - like Lectins ( Siglecs ) Detect Self - Associated Molecular Patterns to Regulate Immune Responses. Cell. Mol. Life Sci. 2020, 77 (4), 593-605. https://doi.org/10.1007/s00018-019-03288-x.

(41) Delaveris, C. S.; Chiu, S. H.; Riley, N. M.; Bertozzi, C. R. Modulation of Immune Cell Reactivity with Cis-Binding Siglec Agonists. Proc. Natt. Acad. Sci. No. In Press.

(42) Rillahan, C. D.; Schwartz, E.; McBride, R.; Fokin, V. V.; Paulson, J. C. Click and Pick: Identification of Sialoside Analogues for Siglec-Based Cell Targeting. Angew. Chemie Int. Ed. 2012, 51 (44), 11014-11018. https://doi.org/10.1002/anie.201205831. 
(43) Hoppenbrouwers, T.; Autar, A. S. A.; Sultan, A. R.; Abraham, T. E.; Van Cappellen, W. A.; Houtsmuller, A. B.; Van Wamel, W. J. B.; Van Beusekom, H. M. M.; Van Neck, J. W.; De Maat, M. P. M. In Vitro Induction of NETosis: Comprehensive Live Imaging Comparison and Systematic Review. PLoS One 2017, 12 (5), 1-29. https://doi.org/10.1371/journal.pone.0176472.

(44) Moreno-Eutimio, M. A.; López-Macías, C.; Pastelin-Palacios, R. Bioinformatic Analysis and Identification of Single-Stranded RNA Sequences Recognized by TLR7/8 in the SARS-CoV-2, SARS-CoV, and MERS-CoV Genomes. Microbes Infect. 2020, 22 (4-5), 226-229. https://doi.org/10.1016/j.micinf.2020.04.009.

(45) Van Der Made, C. I.; Simons, A.; Schuurs-Hoeijmakers, J.; Van Den Heuvel, G.; Mantere, T.; Kersten, S.; Van Deuren, R. C.; Steehouwer, M.; Van Reijmersdal, S. V.; Jaeger, M.; et al. Presence of Genetic Variants among Young Men with Severe COVID-19. J. Am. Med. Assoc. 2020, 324 (7), 663-673. https://doi.org/10.1001/jama.2020.13719.

(46) Gupta, S.; Chan, D. W.; Zaal, K. J.; Kaplan, M. J. A High-Throughput Real-Time Imaging Technique To Quantify NETosis and Distinguish Mechanisms of Cell Death in Human Neutrophils. J. Immunol. 2018, 200 (2), 869-879.

https://doi.org/10.4049/jimmunol.1700905.

(47) Zecha, J.; Satpathy, S.; Kanashova, T.; Avanessian, S. C.; Kane, M. H.; Clauser, K. R.; Mertins, P.; Carr, S. A.; Kuster, B. TMT Labeling for the Masses: A Robust and CostEfficient, in-Solution Labeling Approach. Mol. Cell. Proteomics 2019, 18 (7), 1468-1478. https://doi.org/10.1074/mcp.TIR119.001385.

(48) Lood, C.; Arve, S.; Ledbetter, J.; Elkon, K. B. TLR7 / 8 Activation in Neutrophils Impairs Immune Complex Phagocytosis through Shedding of FcgRIIA. J. Exp. Med. 2017, 214 (7), 2103-2119. 
(49) Zhu, X.; Chen, J. Phosphoproteomic Analyses Provide Insight into Molecular Mechanisms Underlying NETosis. Proteomics 2019, 19, 1900126. https://doi.org/10.1002/pmic.201900126.

(50) Delaveris, C. S.; Webster, E. R.; Banik, S. M.; Boxer, S. G.; Bertozzi, C. R. MembraneTethered Mucin-like Polypeptides Sterically Inhibit Binding and Slow Fusion Kinetics of Influenza A Virus. Proc. Natl. Acad. Sci. 2020, 117 (23), 12643-12650.

(51) Stanczak, M. A.; Zippelius, A.; Läubli, H. Self-Associated Molecular Patterns Mediate Cancer Immune Evasion by Engaging Siglecs on T Cells Graphical Abstract Find the Latest Version : J Clin. Investig. 2018. https://doi.org/10.1172/JCl120612.

(52) Läubli, H.; Pearce, O. M. T.; Schwarz, F.; Siddiqui, S. S.; Deng, L.; Stanczak, M. A. Engagement of Myelomonocytic Siglecs by Tumor-Associated Ligands Modulates the Innate Immune Response to Cancer. 2014, 111 (39), 1-6. https://doi.org/10.1073/pnas.1409580111.

(53) Baskin, J. M.; Wu, X.; Christiano, R.; Oh, M. S.; Schauder, C. M.; Gazzerro, E.; Messa, M.; Baldassari, S.; Assereto, S.; Biancheri, R.; et al. The Leukodystrophy Protein FAM126A (Hyccin) Regulates Ptdlns(4)P Synthesis at the Plasma Membrane. Nat. Cell Biol. 2016, 18 (1). https://doi.org/10.1038/ncb3271.

(54) Phan, T. K.; Williams, S. A.; Bindra, G. K.; Lay, F. T.; Poon, I. K. H.; Hulett, M. D. Phosphoinositides : Multipurpose Cellular Lipids with Emerging Roles in Cell Death. Cell Death Differ. 2019, 26, 781-793. https://doi.org/10.1038/s41418-018-0269-2.

(55) Saito, S.; Kawamura, T.; Higuchi, M.; Kobayashi, T.; Yoshita-takahashi, M.; Yamazaki, M.; Abe, M.; Sakimura, K.; Kanda, Y.; Kawamura, H.; et al. RASAL3, a Novel Hematopoietic RasGAP Protein, Regulates the Number and Functions of NKT Cells. Eur. J. Immunol. 2015, 45, 1512-1523. https://doi.org/10.1002/eji.201444977. 
(56) Moghanloo, E.; Ghorbani, E.; Beikverdi, M. S.; Badameh, P.; Rezaei, S.; Piroozmand, A.; Teimourian, S.; Shahidi, M.; Khorshidi, A. The Netosis Formation of HL-60 Cell Differentiated to Neutrophil-Like Cells by LPS. J. Human, Environ. Heal. Promot. 2018, 4 (3), 138-143. https://doi.org/10.29252/jhehp.4.3.8.

(57) Meizlish, M. L.; Pine, A. B.; Bishai, J. D.; Goshua, G.; Nadelmann, E. R.; Simonov, M.; Chang, C.-H.; Zhang, H.; Shallow, M.; Bahel, P.; et al. A Neutrophil Activation Signature Predicts Critical IIIness and Mortality in COVID-19. medRxiv Prepr. Serv. Heal. Sci. 2020, 06510. https://doi.org/10.1101/2020.09.01.20183897.

(58) Quinn, M. T.; Editors, F. R. D. Neutrophil: Methods and Protocols, Third.; Springer Science: New York, 2020.

(59) Branitzki-Heinemann, K.; Möllerherm, H.; Völlger, L.; Husein, D. M.; de Buhr, N.; Blodkamp, S.; Reuner, F.; Brogden, G.; Naim, H. Y.; Von Köckritz-Blickwede, M. Formation of Neutrophil Extracellular Traps under Low Oxygen Level. Front. Immunol. 2016, 7 (NOV), 1-9. https://doi.org/10.3389/fimmu.2016.00518.

(60) Grasso, S.; Neumann, A.; Lang, I. M.; Etscheid, M.; von Köckritz-Blickwede, M.; Kanse, S. M. Interaction of Factor VII Activating Protease (FSAP) with Neutrophil Extracellular Traps (NETs). Thromb. Res. 2018, 161 (September 2017), 36-42. https://doi.org/10.1016/j.thromres.2017.11.012.

(61) Fuchs, T. A.; Brill, A.; Duerschmied, D.; Schatzberg, D.; Monestier, M.; Myers, D. D.; Wrobleski, S. K.; Wakefield, T. W.; Hartwig, J. H.; Wagner, D. D. Extracellular DNA Traps Promote Thrombosis. Proc. Natl. Acad. Sci. U. S. A. 2010, 107 (36), 15880-15885. https://doi.org/10.1073/pnas.1005743107.

(62) Perdomo, J.; Leung, H. H. L.; Ahmadi, Z.; Yan, F.; Chong, J. J. H.; Passam, F. H.; Chong, B. H. Neutrophil Activation and NETosis Are the Major Drivers of Thrombosis in 
Heparin-Induced Thrombocytopenia. Nat. Commun. 2019, 10 (1), 1-14. https://doi.org/10.1038/s41467-019-09160-7.

(63) Warnatsch, A.; loannou, M.; Wang, Q.; Papayannopoulos, V. Neutrophil Extracellular Traps License Macrophages for Cytokine Production in Atherosclerosis. Science (80-. ). 2015, 349 (6245), 1-6.

(64) Papayannopoulos, V.; Staab, D.; Zychlinsky, A. Neutrophil Elastase Enhances Sputum Solubilization in Cystic Fibrosis Patients Receiving DNase Therapy. 2011, 6 (12), 1-7. https://doi.org/10.1371/journal.pone.0028526.

(65) Büll, C.; Heise, T.; Beurskens, D. M. H.; Riemersma, M.; Ashikov, A.; Rutjes, F. P. J. T.; Van Kuppevelt, T. H.; Lefeber, D. J.; Den Brok, M. H.; Adema, G. J.; et al. Sialic Acid Glycoengineering Using an Unnatural Sialic Acid for the Detection of Sialoglycan Biosynthesis Defects and On-Cell Synthesis of Siglec Ligands. ACS Chem. Biol. 2015, 10 (10), 2353-2363. https://doi.org/10.1021/acschembio.5b00501.

(66) Manda-Handzlik, A.; Bystrzycka, W.; Wachowska, M.; Sieczkowska, S.; StelmaszczykEmmel, A.; Demkow, U.; Ciepiela, O. The Influence of Agents Differentiating HL-60 Cells toward Granulocyte-like Cells on Their Ability to Release Neutrophil Extracellular Traps. Immunol. Cell Biol. 2018, 96 (4), 413-425. https://doi.org/10.1111/imcb.12015.

(67) HaileMariam, M.; Eguez, R. V.; Singh, H.; Bekele, S.; Ameni, G.; Pieper, R.; Yu, Y. STrap, an Ultrafast Sample-Preparation Approach for Shotgun Proteomics. J. Proteome Res. 2018, 17 (9), 2917-2924. https://doi.org/10.1021/acs.jproteome.8b00505.

(68) Brenes, A.; Hukelmann, J.; Bensaddek, D.; Lamond, A. I. Multibatch TMT Reveals False Positives, Batch Effects and Missing Values. Mol. Cell. Proteomics 2019, 18 (10), 19671980. https://doi.org/10.1074/mcp.RA119.001472. 
(69) Hebert, A. S.; Thöing, C.; Riley, N. M.; Kwiecien, N. W.; Shiskova, E.; Huguet, R.;

Cardasis, H. L.; Kuehn, A.; Eliuk, S.; Zabrouskov, V.; et al. Improved Precursor Characterization for Data-Dependent Mass Spectrometry. Anal. Chem. 2018, 90 (3), 2333-2340. https://doi.org/10.1021/acs.analchem.7b04808.

(70) Zhou, H.; Ye, M.; Dong, J.; Corradini, E.; Cristobal, A.; Heck, A. J. R.; Zou, H.;

Mohammed, S. Robust Phosphoproteome Enrichment Using Monodisperse MicrosphereBased Immobilized Titanium (IV) Ion Affinity Chromatography. Nat. Protoc. 2013, 8 (3), 461-480. https://doi.org/10.1038/nprot.2013.010.

(71) Cox, J.; Neuhauser, N.; Michalski, A.; Scheltema, R. A.; Olsen, J. V; Mann, M. Andromeda: A Peptide Search Engine Integrated into the MaxQuant Environment. J. Proteome Res. 2011, 10 (4), 1794-1805. https://doi.org/10.1021/pr101065j.

(72) Tyanova, S.; Temu, T.; Cox, J. The MaxQuant Computational Platform for Mass Spectrometry-Based Shotgun Proteomics. Nat. Protoc. 2016, 11 (12), 2301-2319. https://doi.org/10.1038/nprot.2016.136.

(73) Bateman, A. UniProt: A Worldwide Hub of Protein Knowledge. Nucleic Acids Res. 2019, 47 (D1), D506-D515. https://doi.org/10.1093/nar/gky1049.

(74) Tyanova, S.; Temu, T.; Sinitcyn, P.; Carlson, A.; Hein, M. Y.; Geiger, T.; Mann, M.; Cox, J. The Perseus Computational Platform for Comprehensive Analysis of (Prote)Omics Data. Nat. Methods 2016, 13 (9), 731-740. https://doi.org/10.1038/nmeth.3901.

(75) Perez-Riverol, Y.; Csordas, A.; Bai, J.; Bernal-Llinares, M.; Hewapathirana, S.; Kundu, D. J.; Inuganti, A.; Griss, J.; Mayer, G.; Eisenacher, M.; et al. The PRIDE Database and Related Tools and Resources in 2019: Improving Support for Quantification Data. Nucleic Acids Res. 2019, 47 (D1), D442-D450. https://doi.org/10.1093/nar/gky1106. 
591 (76) Stuart, T.; Butler, A.; Hoffman, P.; Hafemeister, C.; Papalexi, E.; Mauck, W. M.; Hao, Y.;

592 Stoeckius, M.; Smibert, P.; Satija, R. Comprehensive Integration of Single-Cell Data. Cell

593 2019, 177 (7), 1888-1902.e21. https://doi.org/10.1016/j.cell.2019.05.031.

594

595 


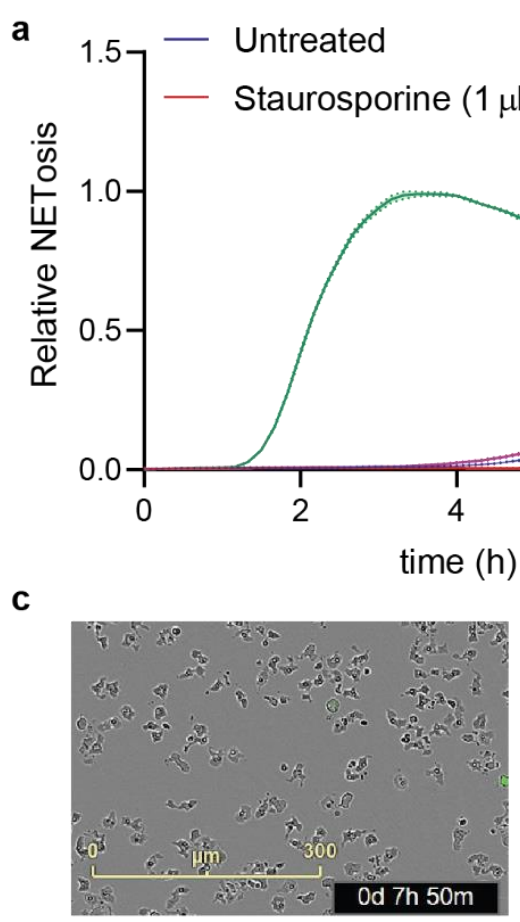

staurosporine (apoptosis)

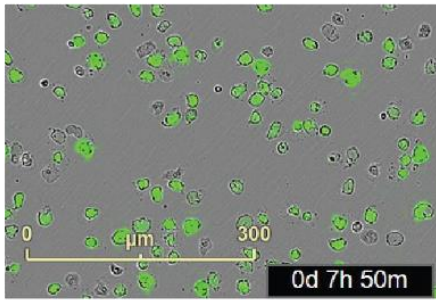

PMA

(classic NETosis)
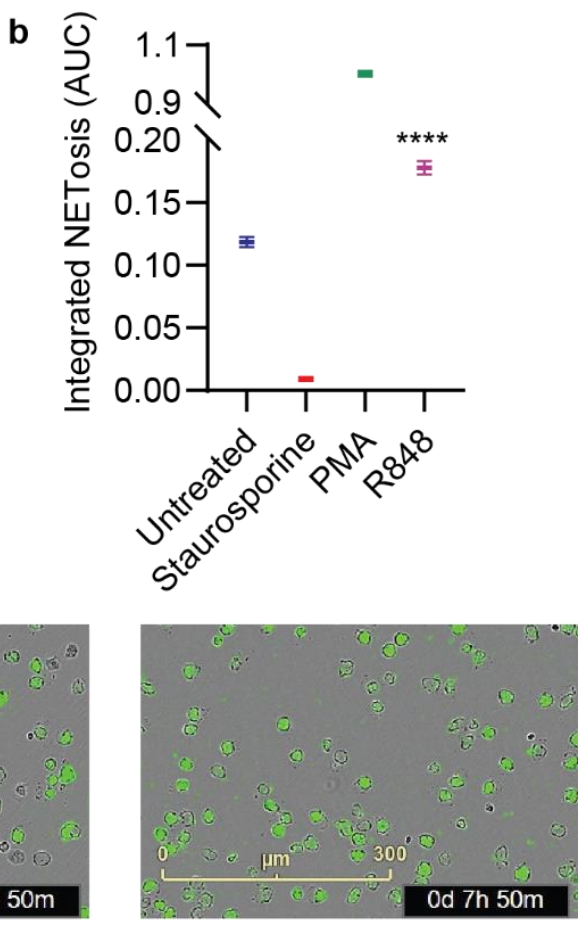

R848

(viral NETosis)

Figure S1.TLR-7/8 agonist R848 induces NETosis in primary neutrophils. (a-c) Primary neutrophils were treated with compounds at the concentrations detailed in (a) in IMDM supplemented $0.5 \%$ hiFBS containing the membrane impermeable DNA intercalator Cytotox

602 Green (250 nM). Images were acquired by fluorescence microscopy every $10 \mathrm{~min}$ for $8 \mathrm{~h}$. The area of all green fluorescent objects $>300 \mu \mathrm{m}^{2}$ was quantified and averaged across three images per well. Relative NETosis was determined by normalizing to the maximal NET area from PMA treatment $(\mathrm{t}=3 \mathrm{~h})$. (a) Time-course data for NET formation and degradation over time. Error bands represent SEM. (b) Quantitation of (a) as area under the curve. Error bars represent SD. All data are representative of multiple independent experiments using neutrophils from different donors. ${ }^{* * * *} \mathrm{p}<0.0001$. (c) Representative images of neutrophils treated with staurosporine, PMA, or R848 stained with Cytotox Green at $t=7 \mathrm{~h} 50 \mathrm{~m}$. 
a

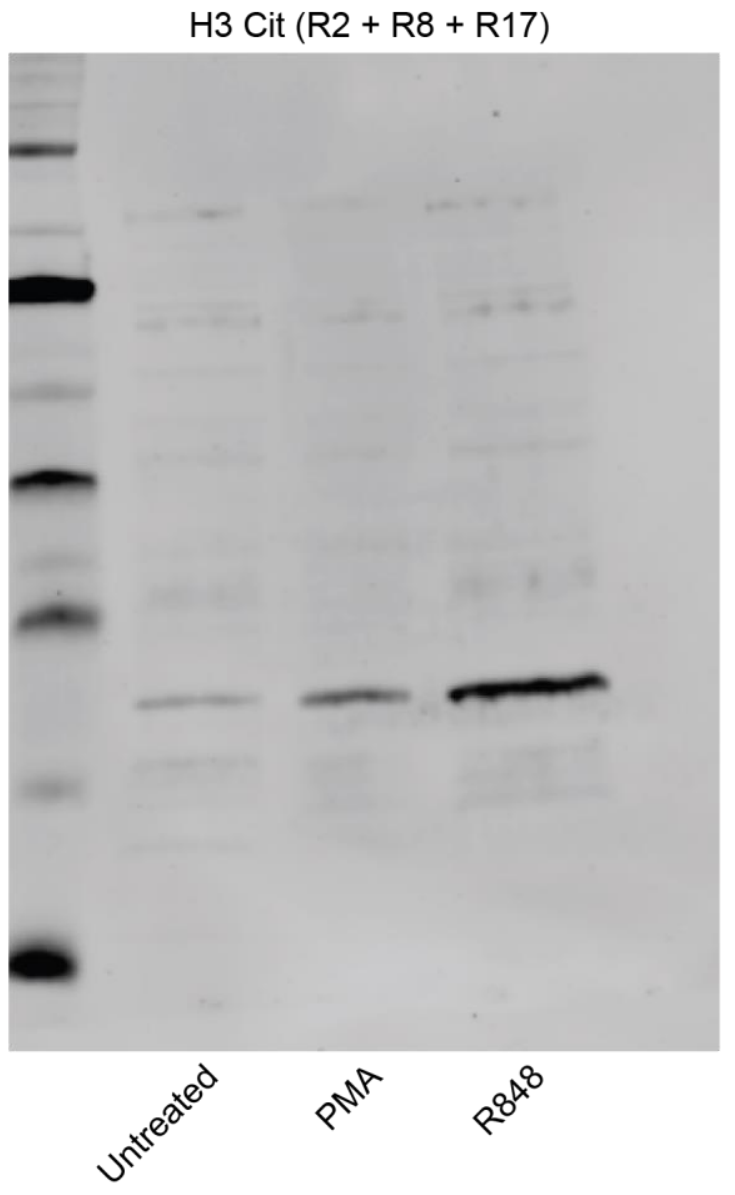

b

GAPDH

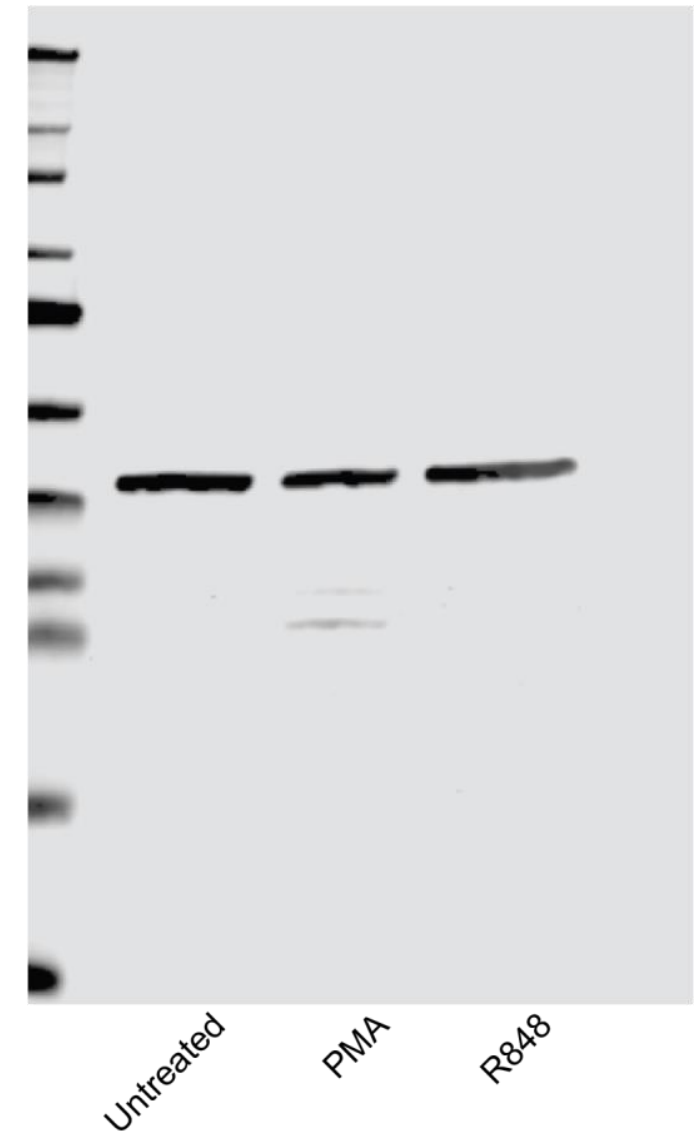

613 Figure S2. R848 induces rapid citrullination of H3 in primary neutrophils. Primary neutrophils

614 were treated with compound in IMDM [+] 0.5\% hiFBS for $30 \mathrm{~min}$ before being lysed and

615 analyzed by Western blot for citrullination of histone H3. (a) H3Cit (R2 + R8 + R17). (b) GAPDH. 
a

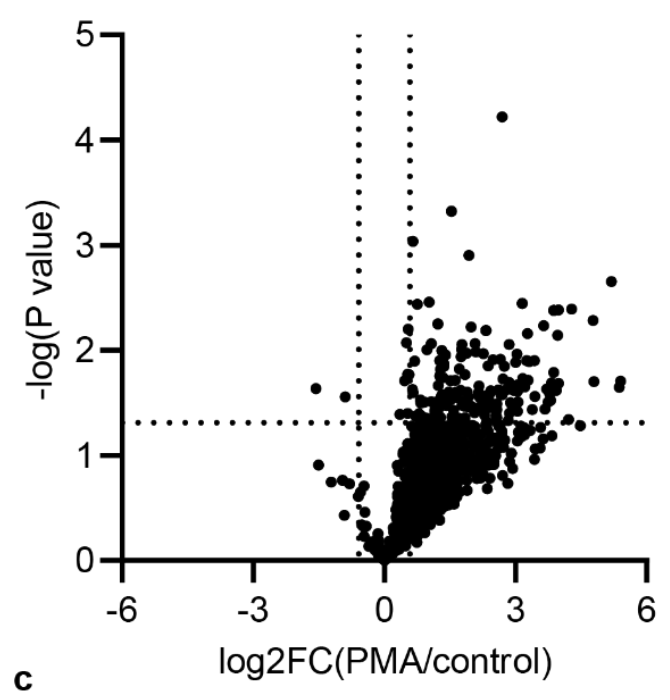

C

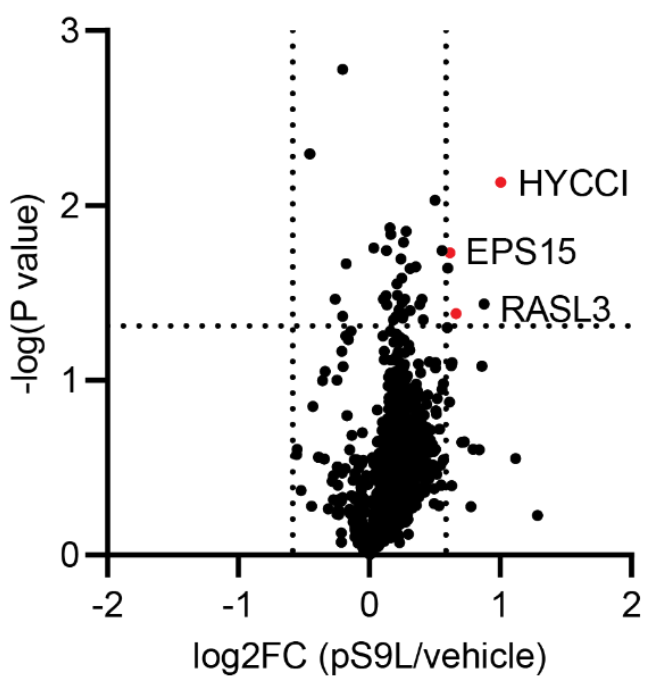

b
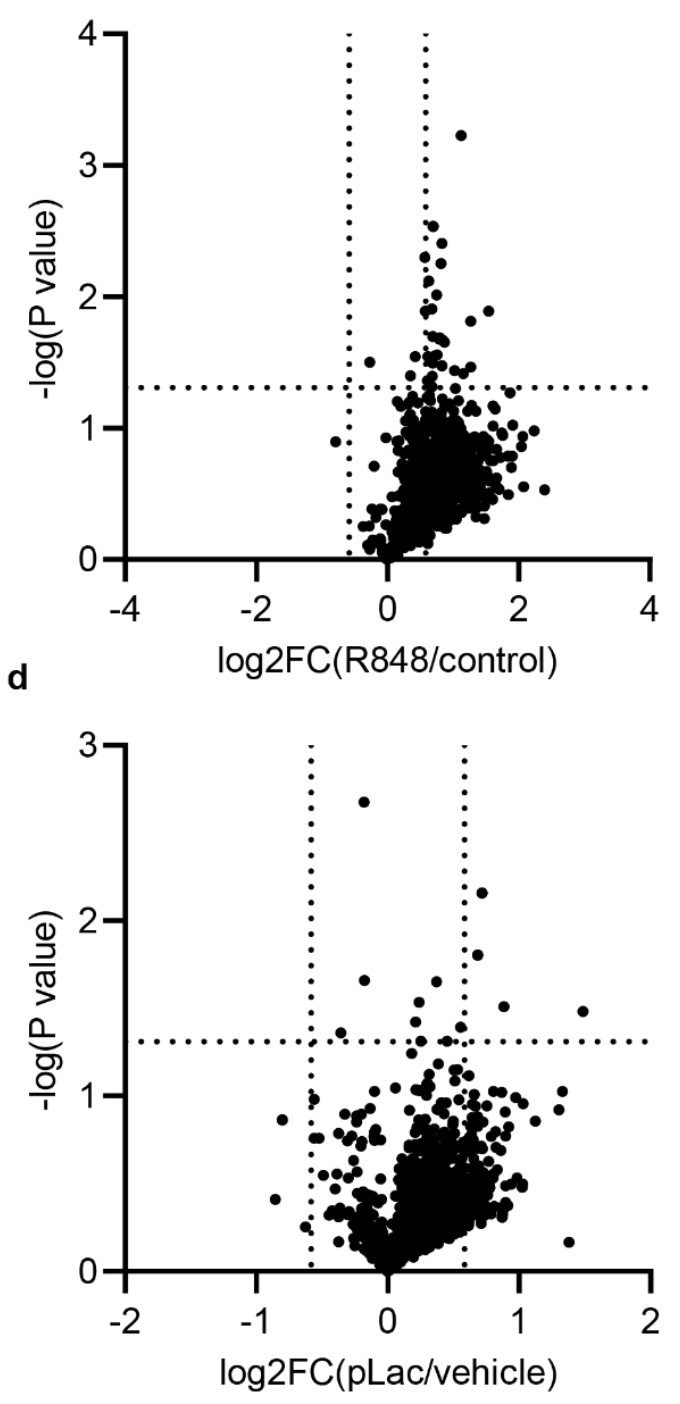

618

619

620

621

622

623

624

625

Figure S3. Volcano plots of significance vs. fold-change for phosphopeptides enriched from neutrophil lysates. Neutrophils isolated from healthy donor whole blood were treated with either PMA (500 nM) or R848 $(10 \mu \mathrm{M})$ or left untreated for 30 min $(\mathbf{a}, \mathbf{b})$ or with $\mathrm{R} 848(10 \mu \mathrm{M})$ and one of pS9L (500 nM), pLac (500 nM) or vehicle for $15 \mathrm{~min}(\mathbf{c}, \mathbf{d})$ in IMDM [+] 0.5\% hiFBS. The cells were lysed, digested, and phosphopeptides were enriched before being TMT-labeled and analyzed by LC-MS. Vertical lines represent $x=+/-0.585$ (corresponding to 1.5 -fold change) and horizontal lines represent $p=0.05$ as determined by a paired t-test to account for variability between neutrophil donors. Each datapoint represents the average fold-change from three 
626 different donors. (a) Fold-change of PMA compared to untreated neutrophils. (b) Fold-change of 627 R848 compared to untreated neutrophils. (c) Fold-change of pS9L-treated compared to vehicle628 treated R848-stimualted neutrophils. Red dots highlight significant hits unique to this dataset 629 compared to (d). (d) Fold-change of pLac-treated compared to vehicle-treated R848-stimualted 630 neutrophils.

631 


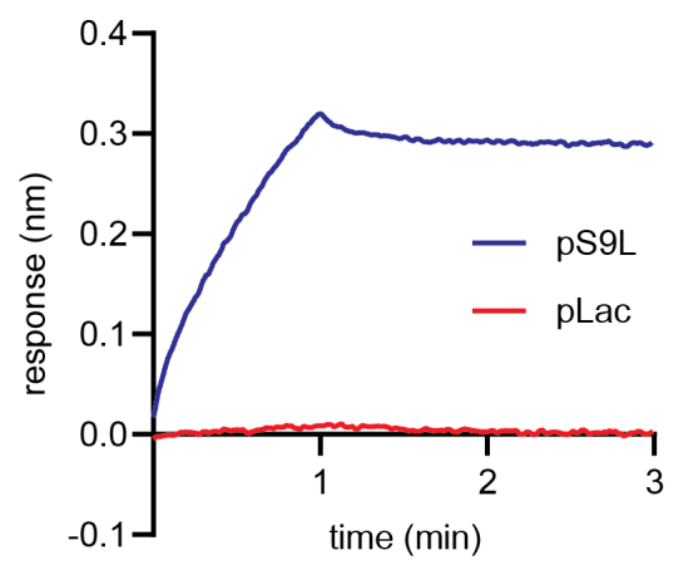

633 Figure S4. Siglec-9 binds pS9L but not pLac. Siglec-9-Fc was immobilized onto anti-hFc-coated 634 tips on an OctetRed96 to a threshold of $0.4 \mathrm{~nm}$. Association of glycopolypeptide $(2.5 \mu \mathrm{M})$ was 635 measured by dipping Siglec-9-Fc coated tips into a solution of glycopolypeptide in PBS with $6360.1 \%$ BSA to abrogate nonspecific binding. Tips were conditioned prior to the first assay and 637 regenerated between runs with three washes in $100 \mathrm{mM}$ glycine buffer (pH 1.5). 


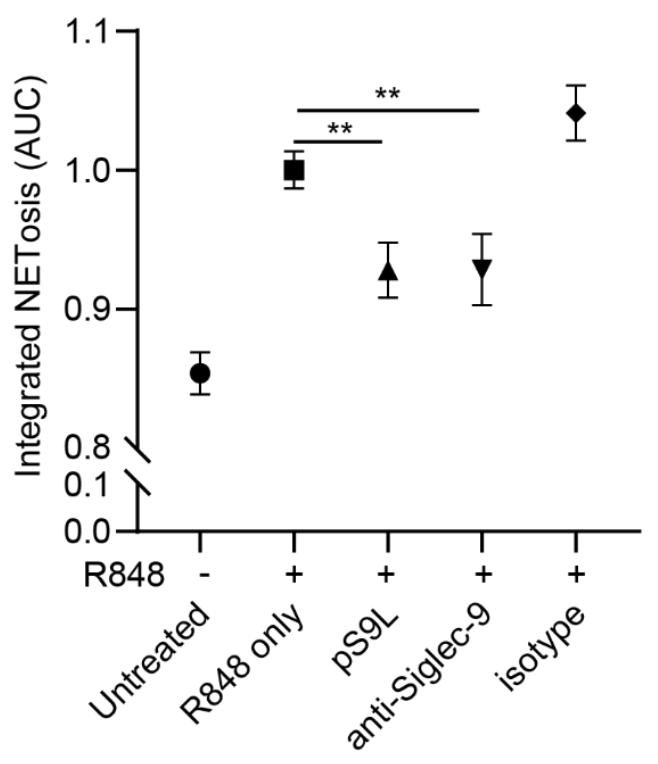

640 Figure S5. pS9L inhibits NETosis comparably to Siglec-9 agonist antibody. Primary neutrophils 641 were cotreated with R848 $(10 \mu \mathrm{M})$ and either cis-binding Siglec-9 agonist pS9L (500 nM) or 642 antibody (anti-Siglec-9 clone 191240 or an isotype control $\mathrm{lgG}$ ) (35 $\mu \mathrm{g} / \mathrm{mL}$ ) precomplexed with 643 Protein A $(5 \mu \mathrm{g} / \mathrm{mL})$ or vehicle in IMDM supplemented with $0.5 \%$ hiFBS and containing Cytotox 644 Green (250 nM). Images were acquired by fluorescence microscopy on an Incucyte Zoom every $6451 \mathrm{~h}$ for $12 \mathrm{~h}$. The area of all green fluorescent objects $>200 \mu \mathrm{m}^{2}$ was quantified and averaged 646 across three images per well. The area under the curve was quantified and normalized to the 647 mean value for R848 treated neutrophils with no cotreatment. 

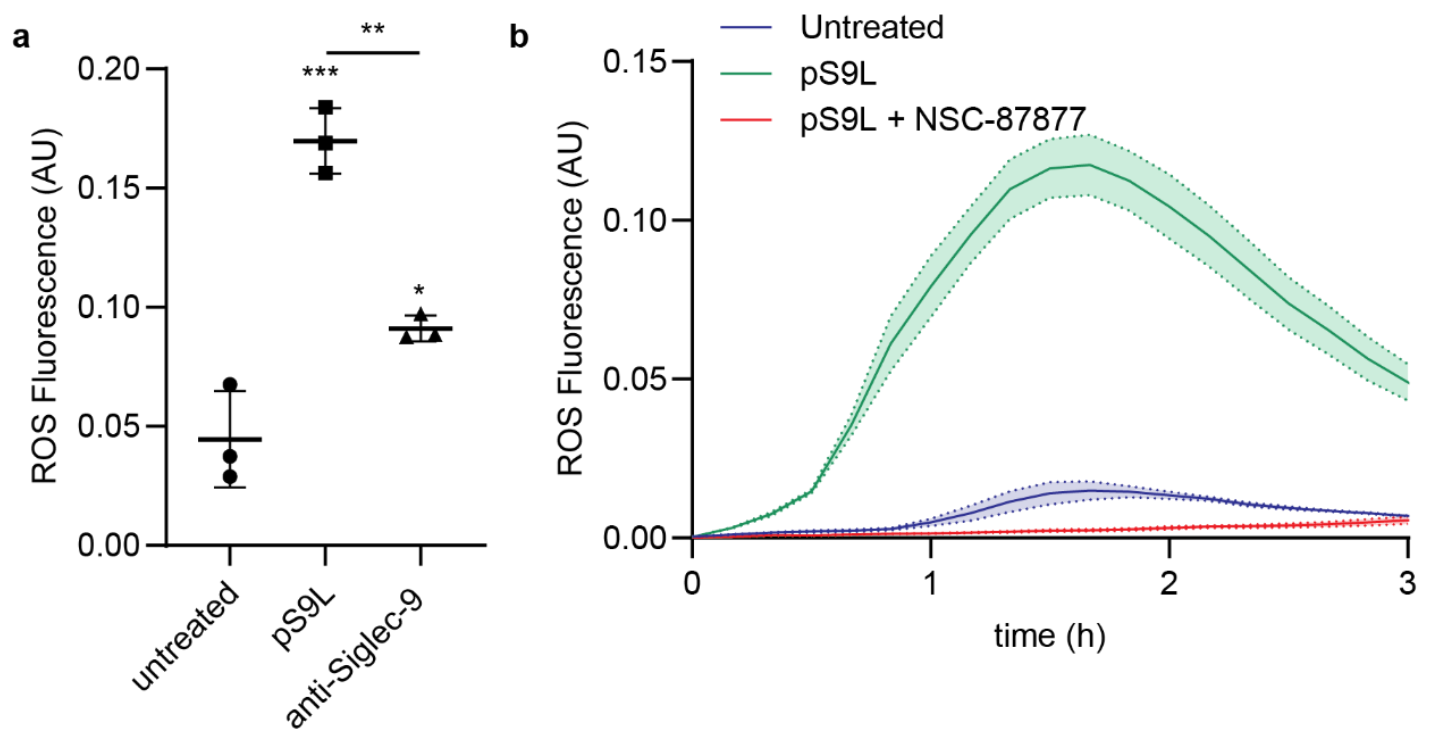

650 Figure S6. Siglec-9 engagement induces a SHP-1/2 dependent oxidative burst. (a) Primary

651 neutrophils were treated with either cis-binding Siglec-9 agonist pS9L (500 nM) or anti-Siglec-9

652 (clone 191240) $(35 \mu \mathrm{g} / \mathrm{mL})$ precomplexed with Protein $\mathrm{A}(5 \mu \mathrm{g} / \mathrm{mL})$ or vehicle in IMDM

653 supplemented with $0.5 \%$ hiFBS and containing CellROX Deep Red $(5 \mu \mathrm{M})$. Phase and red

654 fluorescence images were acquired every 10 min using an Incucyte $\mathrm{S} 3$ in a $37^{\circ} \mathrm{C}$ and $5 \% \mathrm{CO}_{2}$

655 incubator. ROS fluorescence was quantitated using integrated intensity normalized to

656 confluence. Statistics were determined by one-way ANOVA. ${ }^{*}=p<0.05 ;{ }^{* *}=p<0.01 ;{ }^{* * *}=p<$

657 0.001. (b) As in (a), with or without the SHP-1/2 inhibitor NSC-87877 $(50 \mu \mathrm{M})$, as has been

658 previously used to study SHP-1/2-mediated Siglec activity. ${ }^{65}$ 


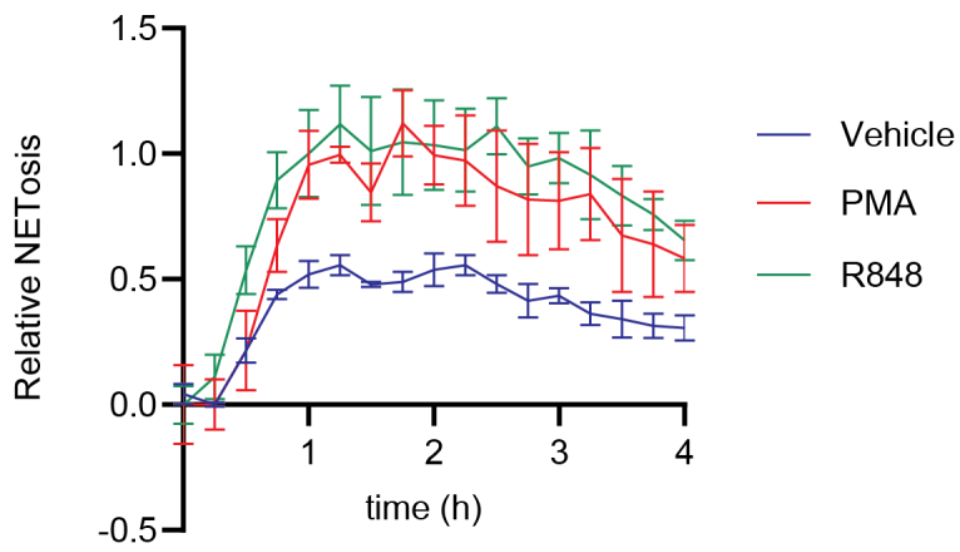

661 Figure S7. TLR-7/8 agonist R848 induces NETosis in dHL-60 cells. HL-60 cells were cultured in

662 RPMI-1640 supplemented with $20 \%$ hiFBS in the presence of DMSO $(1.25 \% \mathrm{v} / \mathrm{v})$ and ATRA

$663(100 \mathrm{nM})$ for $6 \mathrm{~d}$. Cells were then seeded in serum-free RPMI-1640 containing Cytotox Green

664 (250 nM) and NETosis was induced with PMA (100 nM), R848 (10 $\mu \mathrm{M})$, or vehicle. Phase and 665 green fluorescence images were acquired every 15 min using an Incucyte S3. The area of all 666 green fluorescent objects $>200 \mu \mathrm{m}^{2}$ was quantified and averaged across three images per well.

667 Relative NETosis was determined by normalizing to the maximal NET area from PMA treatment 668 alone $(\mathrm{t}=2.5 \mathrm{~h})$. 


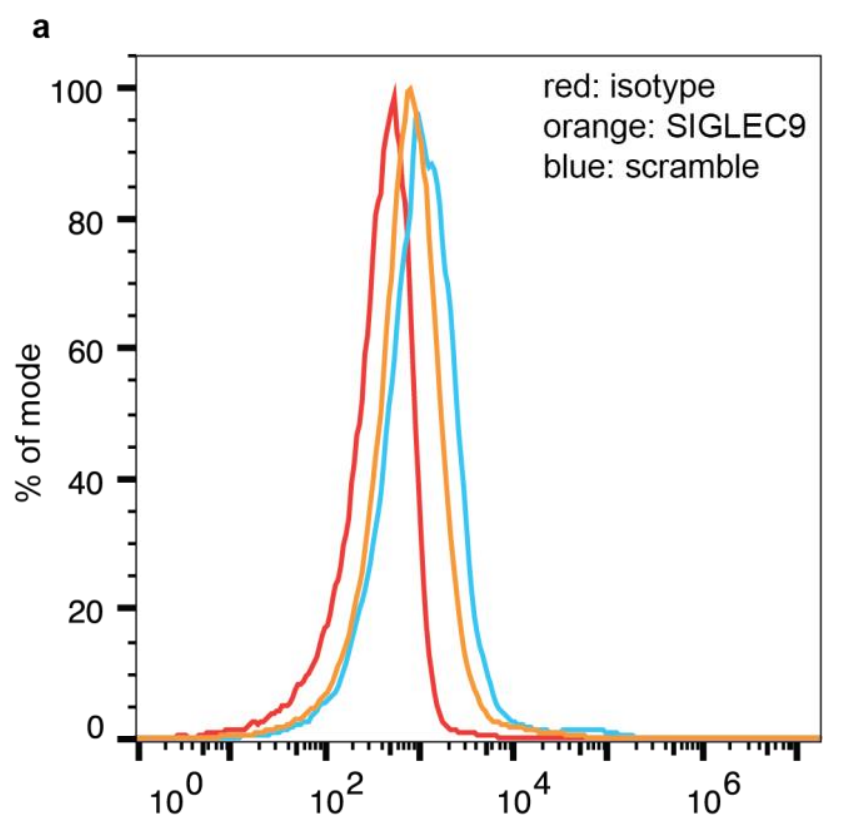

b

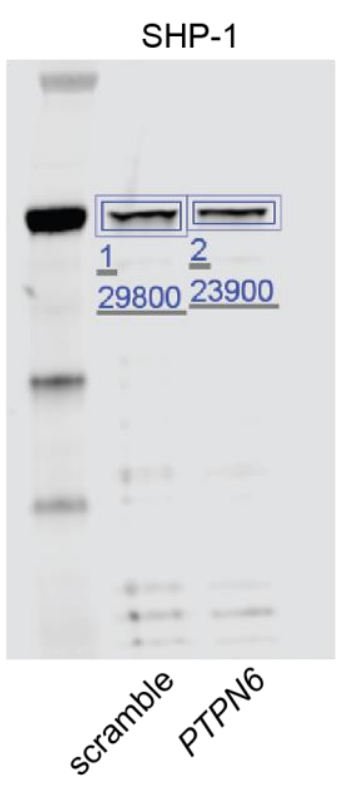

C

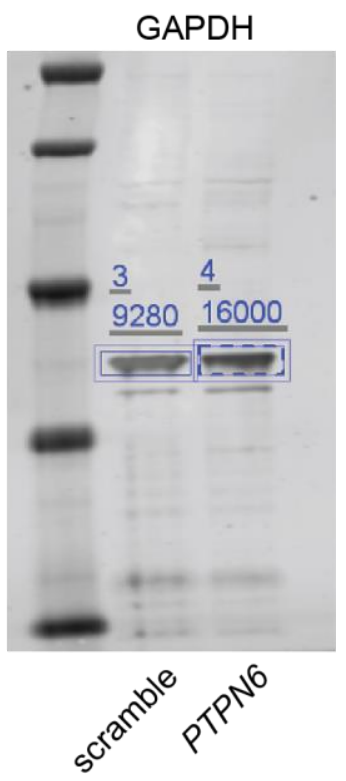

Anti-Siglec-9-647 Fluorescence (AU)

671 Figure S8. Siglec-9 and SHP-1 levels are reduced by siRNA knock-down of differentiated HL-

67260 neutrophil-like cells. HL-60 cells were cultured in RPMI-1640 supplemented with $20 \%$ hiFBS

673 in the presence of DMSO (1.25\% v/v) and ATRA (100 nM). After $4 \mathrm{~d}$, the media was changed

674 and cells were treated with siRNA's. On day 6, expression of target proteins was assayed. (a)

675 dHL-60's treated with either SIGLEC9-targeting or scramble siRNA's were stained with an anti-

676 Siglec-9 antibody (clone K8, AlexaFluor647 conjugate) or an isotype control and analyzed by

677 flow cytometry. (b,c) Lysates from dHL-60's treated with siRNA's targeting PTPN6 (encoding

678 SHP-1) or a scrambled negative control were analyzed by Western blot, staining for either SHP-

6791 (b) or GAPDH (c). Fluorescence signal was quantitated by LiCOR. 

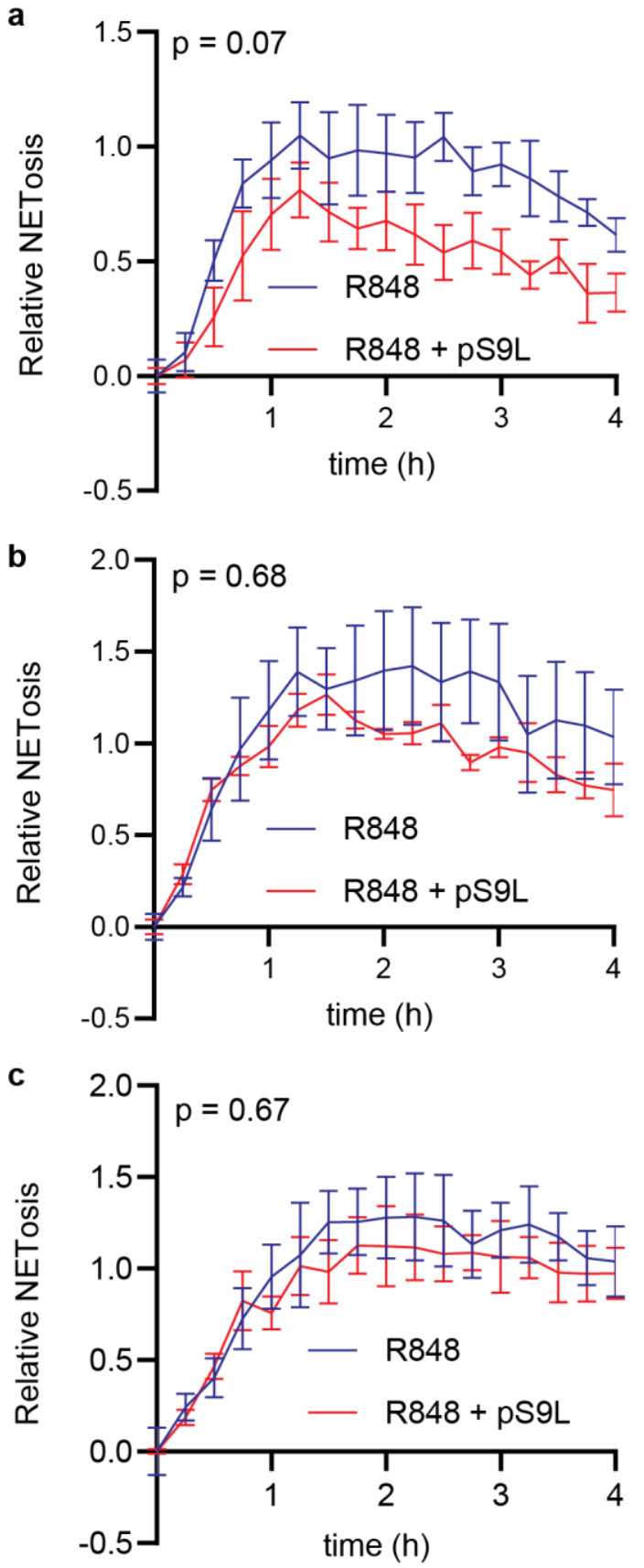

681 Figure S9. Cis Siglec-9 agonist pS9L inhibits NETosis via Siglec-9 and SHP-1. HL-60 cells 682 were cultured in RPMI-1640 supplemented with $20 \%$ hiFBS in the presence of DMSO $(1.25 \%$ $683 \mathrm{v} / \mathrm{v})$ and ATRA (100 nM). After $4 \mathrm{~d}$, the media was changed and cells were treated with siRNA's. 684 On day 6, cells were then seeded in serum-free RPMI-1640 containing Cytotox Green (250 nM) 685 and NETosis was induced with R848 $(10 \mu \mathrm{M})$ with or without pS9L (500 nM). Phase and green 
686 fluorescence images were acquired every 15 min using an Incucyte S3. The area of all green 687 fluorescent objects $>200 \mu \mathrm{m}^{2}$ was quantified and averaged across three images per well.

688 Relative NETosis was determined by normalizing to the maximal NET area from PMA treatment 689 alone $(\mathrm{t}=2.5 \mathrm{~h})$. (a) Scramble siRNA control. (b) SIGLEC9 targeting siRNA cocktail. (c) PTPN6 690 siRNA. Statistics were calculated by two-way ANOVA.

691 


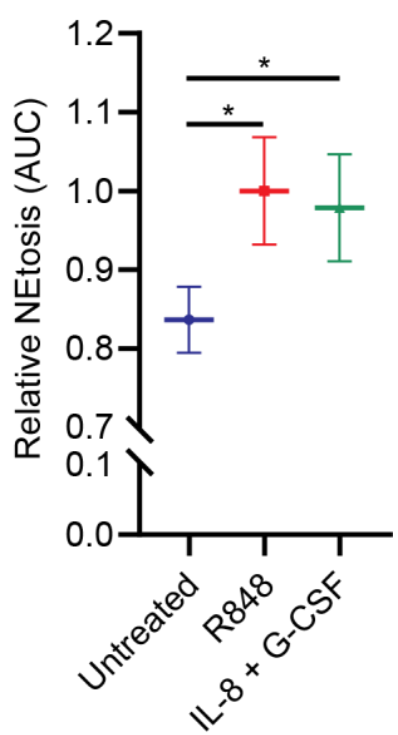

693 Figure S10. A combination of IL-8 and G-CSF induce NETosis in primary neutrophils. Primary 694 neutrophils were stimulated with R848 (10 $\mu \mathrm{M})$, a combination of IL-8 (100 ng/mL) and G-CSF $695(100 \mathrm{ng} / \mathrm{mL})$, or vehicle and cultured in IMDM supplemented $0.5 \%$ hiFBS containing the 696 membrane impermeable DNA intercalator Cytotox Green (250 nM). Images were acquired by 697 fluorescence microscopy every $1 \mathrm{~h}$ for $8 \mathrm{~h}$. The area of all green fluorescent objects $>300 \mu \mathrm{m}^{2}$ 698 was quantified and averaged across three images per well. Data were quantitated as area 699 under the curve measurements. Error bars represent SD. Data are representative of multiple 700 independent experiments using neutrophils from different donors. * $p<0.05$. 
a

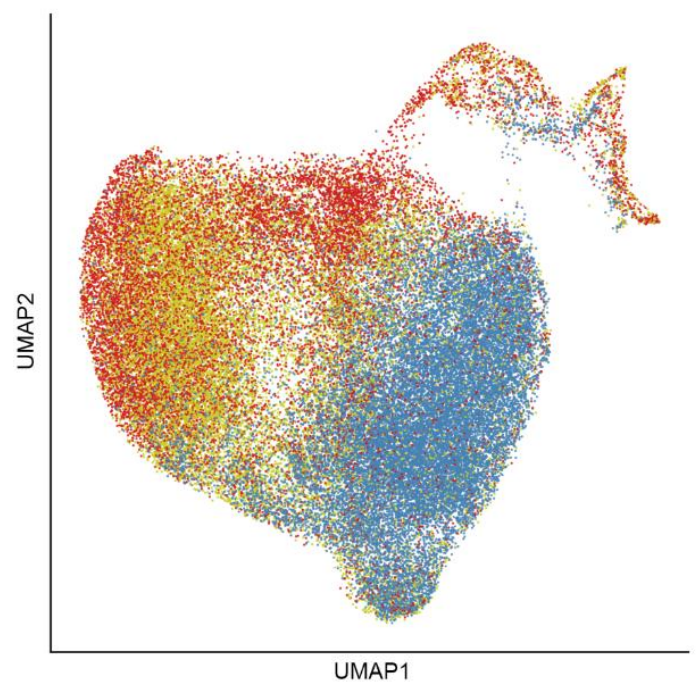

C

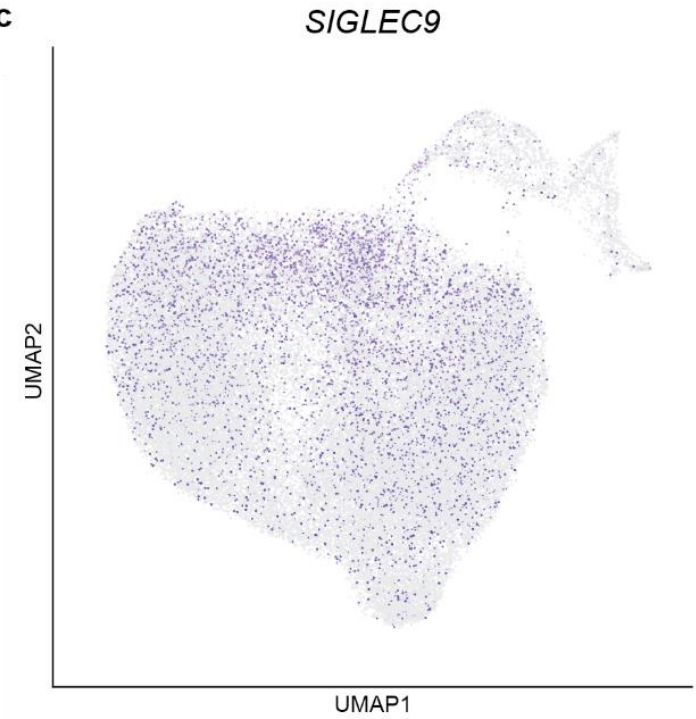

b

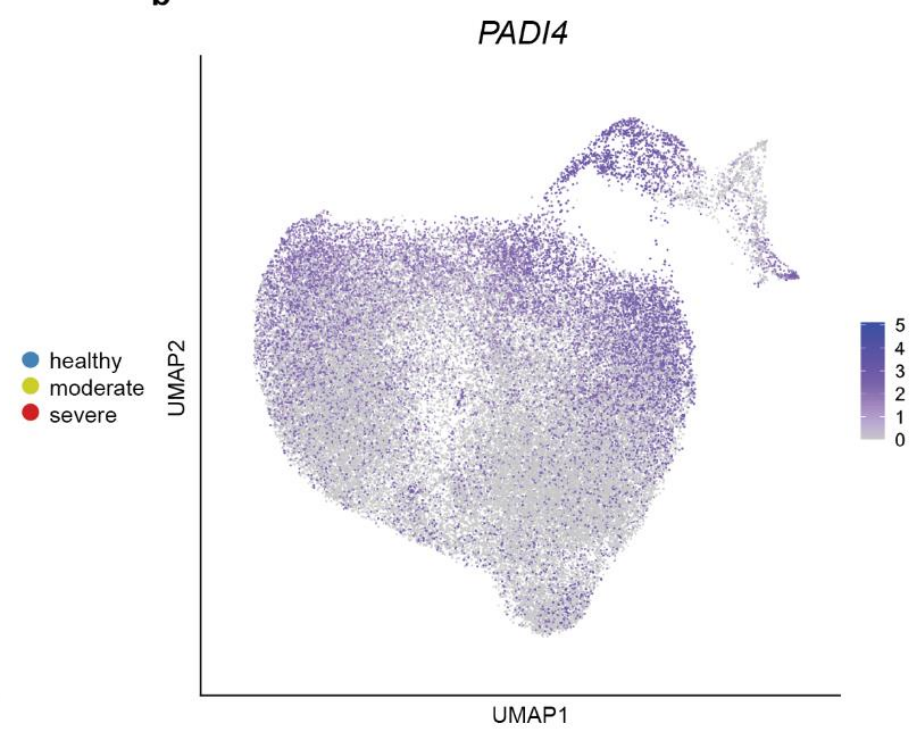

d
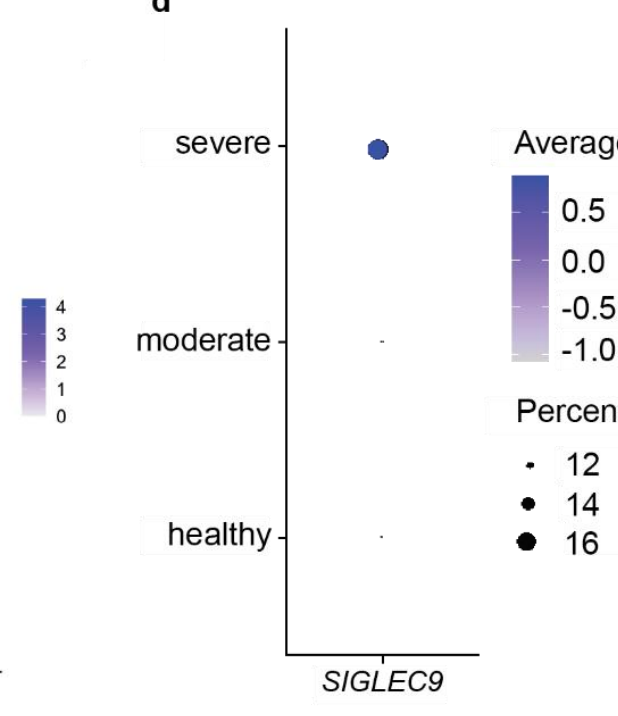

701

702 Figure S11. SIGLEC9 and PADI4 are upregulated by neutrophils of patients with severe COVID-19. (a-c) Uniform Manifold Approximation and Projection (UMAP) plot of neutrophils from the single-cell transcriptomic dataset published by Schulte-Schrepping and coworkers. ${ }^{8}$ (a) Each cell is colored by the WHO severity score class (moderate, WHO score 4-5; severe, WHO score 6-8) of the patient at the time of sample collection, demonstrating strong severity-

707 associated phenotypic reconfiguration of neutrophil transcriptome in COVID-19. (b) Each cell is colored by relative PADI4 expression. (c) Each cell is colored by relative SIGLEC9 expression. 
709 (d) Dot plot depicting average and percent SIGLEC9 expression by neutrophils in each WHO 710 severity score class, indicating upregulation of SIGLEC9 in severe COVID-19. 


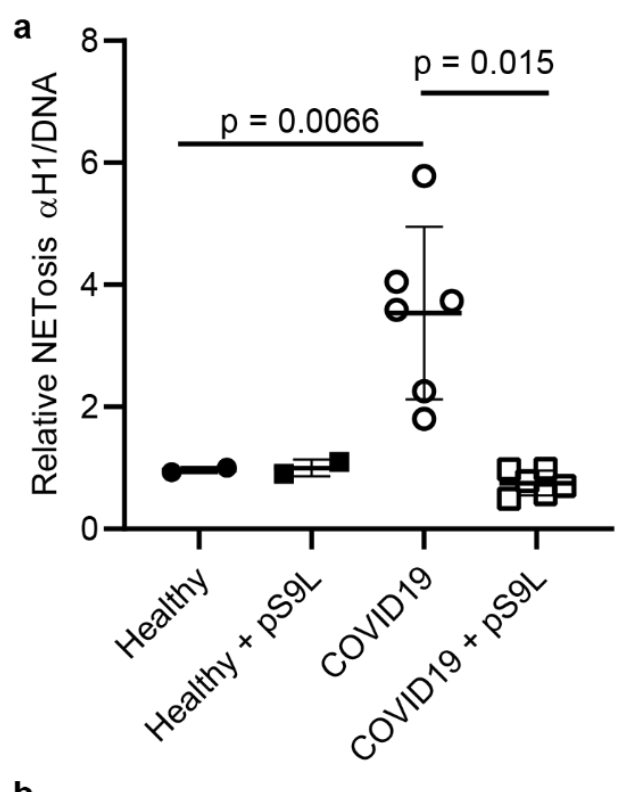

b

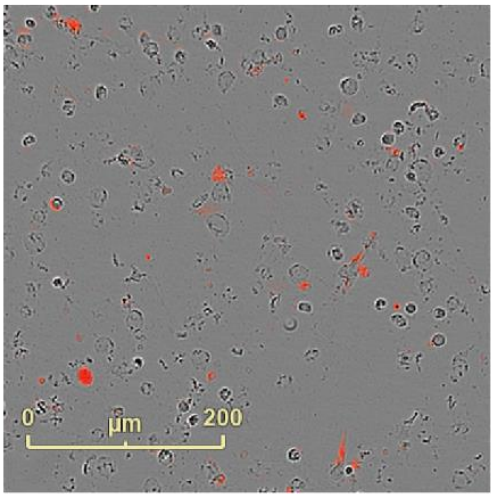

$10 \%$ healthy plasma

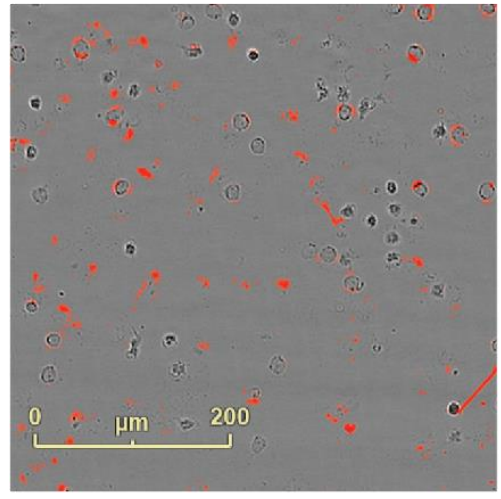

$10 \%$ COVID plasma

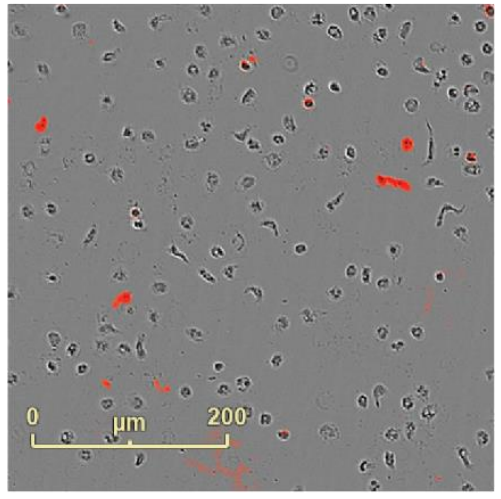

$10 \%$ COVID plasma + pS9L

712 Figure S12. A Siglec-9 agonist inhibits NETosis of neutrophils induced by $10 \%$ COVID-19

713 plasma diluted in media. Neutrophils were isolated from healthy donors and cultured with pS9L

$714(500 \mathrm{nM})$ in undiluted plasma for $4 \mathrm{~h}$. Plasma was citrate anti-coagulated and from healthy

715 donors or COVID-19 patients and diluted to 10\% in IMDM. Cells were then fixed with $4 \%$

716 paraformaldehyde and blocked in 10\% goat serum without permeabilization. (a) NETs were

717 detected by immunocytochemistry with a mouse anti-H1/DNA complex primary antibody

718 (MAB3864, 1:100) and a goat anti-mouse AlexaFluor594 (1:1000) secondary. Cells were

719 counterstained with MemGlow488 prior to imaging but after immunostaining. The area of all red

720 fluorescent objects $>25 \mu \mathrm{m}^{2}$ and $>2.0 \mathrm{RCU}$ was quantified and averaged across four images per 
721 well for three wells and normalized to cell count as determined by green fluorescent objects $>50$

$722 \mu \mathrm{m}^{2}$ and $>10.0 \mathrm{GCU}$. Each data point represents the mean value from technical replicates of an 723 individual donor/patient. Error bars represent SD. Statistics were determined using a mixed 724 effects model to account for differences in neutrophil donors and paired for matched patient 725 plasmas. (b) Representative images showing anti-H1/DNA staining.

726

727 
a

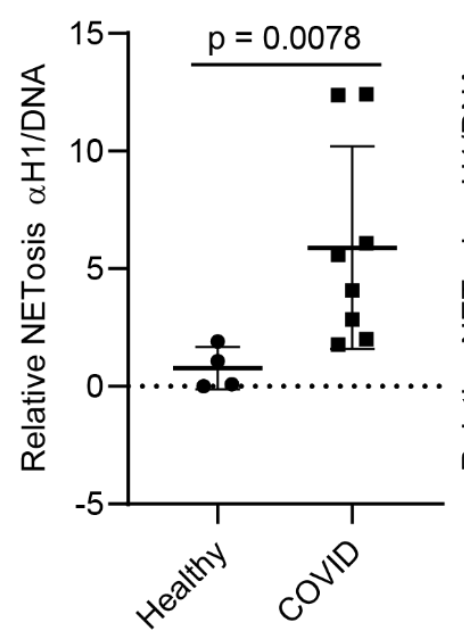

b

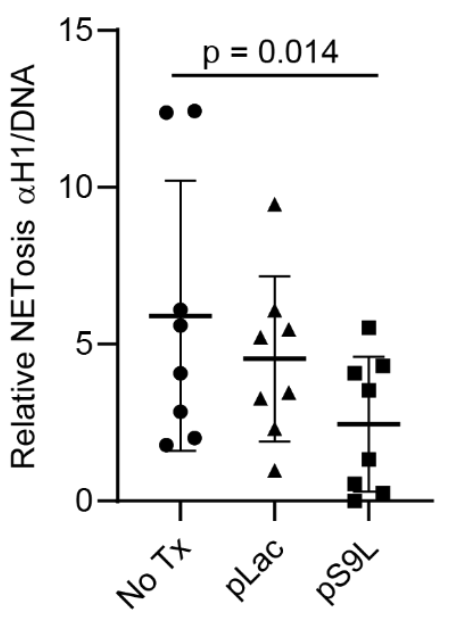

C
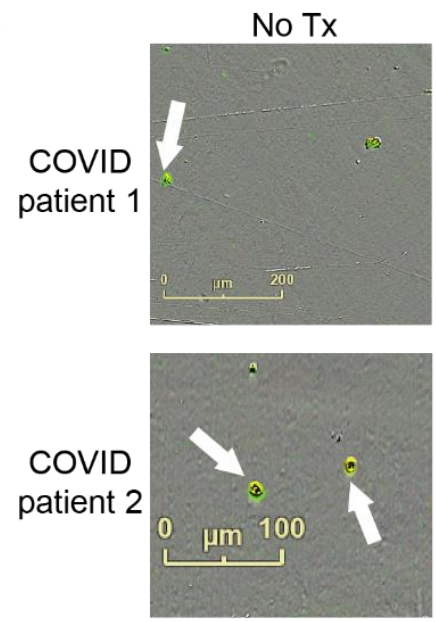

pS9L

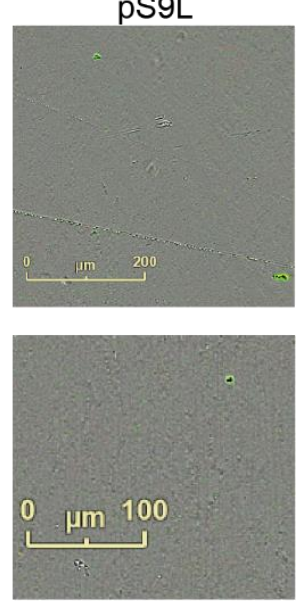

Figure S13. A Siglec-9 agonist inhibits NETosis of neutrophils induced by undiluted COVID-19 plasma. Neutrophils were isolated from healthy donors and cultured with pS9L (500 nM) or pLac (500 nM) in undiluted plasma for $4 \mathrm{~h}$. Plasma was citrate anti-coagulated and from healthy donors or COVID-19 patients. Cells were then fixed with $4 \%$ paraformaldehyde and blocked in $10 \%$ goat serum without permeabilization. (a) NETs were detected by immunocytochemistry with a mouse anti-H1/DNA complex primary antibody (MAB3864, 1:100) and a goat anti-mouse AlexaFluor594 (1:1000) secondary. Cells were counterstained with MemGlow488 prior to imaging but after immunostaining. The area of all red fluorescent objects $>25 \mu \mathrm{m}^{2}$ and $>2.0$ RCU was quantified and averaged across four images per well for three wells and normalized to cell count as determined by green fluorescent objects $>50 \mu \mathrm{m}^{2}$ and $>10.0 \mathrm{GCU}$. Each data point represents the mean value from technical replicates of an individual donor/patient. Error bars represent SD. Statistics were determined using a mixed effects model to account for differences

742 in neutrophil donors and paired for matched patient plasmas. (b) Representative images

743 showing anti-H1/DNA staining (red) overlayed with MemGlow488 (green). 


\section{Glycopolypeptide synthesis.}

749 Glycopolypeptides were synthesized as previously described. ${ }^{41,50}$ In brief, $\mathrm{N}$-carboxyanhydride 750 monomers were polymerized either with a functionalized initiator that affords a membrane751 tethering moiety or with a $\mathrm{Ni}(0)$ initiator that affords a soluble polypeptide. Polypeptides were 752 deprotected with hydrazine monohydrate and purified by dialysis. In some cases, lactose753 bearing scaffolds were then chemoenzymatically functionalized to afford terminal Siglec- 9 754 ligands. ${ }^{41}$ All glycopolypeptides were endotoxin purified on Pierce endotoxin removal resin 755 (ThermoFisher, 88274) and sterile filtered $(0.22 \mu \mathrm{m})$ before use.

757 Primary cell isolation.

758 Whole blood samples were obtained from the healthy donors and anti-coagulated with K2EDTA 759 at the Stanford Blood Center. Samples were de-identified by the Stanford Blood Center.

760 Neutrophils were purified on the same day as isolation, and the whole blood samples were kept 761 at room temperature between collection and neutrophil isolation. Neutrophils were isolated by 762 EasySep Direct Neutrophil Isolation Kit (StemCell Technologies, 19666) according to the 763 manufacturer's instructions in $5 \mathrm{~mL}$ aliquots on a magnetized rack (StemCell Technologies, 764 18103). Efficiency of isolation was determined by flow cytometry to identify CD45 and CD14 hi 765 cells. Neutrophils were routinely obtained in $>96 \%$ purity via this method.

767 COVID-19 patients and specimen collection.

768 Peripheral blood was collected from patients enrolled in the IRB-approved Stanford University 769 Emergency Department COVID-19 Biobank beginning in April 2020 after written informed 
consent from patients or their surrogates. Eligibility criteria included age $\geq 18$ years and presentation to the Stanford Hospital with a positive SARS-CoV-2 nasopharyngeal swab by RTPCR. Patients were phenotyped for COVID-19 severity according to the universal World Health Organization (WHO) ordinal scale. Blood draws from patients occurred upon presentation to the Stanford Hospital Emergency Department in concert with usual care to avoid unnecessary personal protective equipment usage. Blood was collected into CPT vacutainers (Becton, Dickinson, and Co.) and plasma isolated, aliquoted, and stored at $-80^{\circ} \mathrm{C}$ after centrifugation at $1800 \times \mathrm{g}$ for 20 minutes at $25^{\circ} \mathrm{C}$. All sample processing occurred under BSL2+ biosafety precautions as approved by Stanford University APB.

\section{Cell culture.}

The acute promyelocytic leukemia cell line HL-60 (ATCC, CCL-240) were cultured in RPMI1640 supplemented with $20 \%$ hiFBS (ThermoFisher, 10-438-026). Cultures were thoroughly washed with prewarmed complete media when bringing out of cryostorage to completely remove DMSO, as residual DMSO can induce differentiation in HL-60s. ${ }^{66}$ Cells were allowed to recover for at least two weeks prior to any NETosis experiments. Cultures were not allowed to exceed a density of $5 \mathrm{e} 5$ cells per $\mathrm{mL}$ of culture media, as high densities can also induce differentiation. ${ }^{66}$

To induce differentiation of HL-60's, 2e6 cells were harvested by centrifugation (300 rcf, 5 min) and resuspended in complete media (RPMI-1640 +20\% hiFBS) supplemented with 100 nM ATRA (Sigma-Aldrich, R2625) and DMSO (Sigma-Aldrich, D8414). Cells were cultured for 5-6 days before use, with a complete media change $48 \mathrm{~h}$ prior to use. 
795 For live cell NETosis assays were performed in 96 well plates (Corning, 3595) and monitored by 796 fluorescence microscopy with an Incucyte S3 (Essen Biosciences) or an Incucyte ZOOM (Essen 797 Biosciences). DNA staining was performed using membrane impermeable fluorogenic DNA 798 intercalators Cytotox Green (Essen Biosciences, 4633) or Cytotox Red (Essen Biosciences, 799 4632).

Plates were prepared containing 10X solutions of compounds $(20 \mu \mathrm{L})$ and a $2 \mathrm{X}$ solution of DNA dye $(100 \mu \mathrm{L})$ in serum-free media (IMDM for primary neutrophils, RPMI-1640 for HL-60s) lacking phenol red. Freshly harvested neutrophils in media (IMDM with 1.2\% hiFBS for primary neutrophils for $0.5 \%$ final concentration of hiFBS, serum free RPMI-1640 for HL-60s) were then added to the plate $(80 \mu \mathrm{L}, 2.5 \mathrm{e} 5$ per $\mathrm{mL}, 2 \mathrm{e} 4$ per well) from a cell suspension. The plates were briefly centrifuged at 300 rcf for 1 min to settle the cell suspension, and the plates were immediately transferred to the Incucyte for periodic monitoring (every 10 min for $8 \mathrm{~h}$ or every 15 $\min$ for $12 \mathrm{~h})$.

For NETosis assays with diluted COVID-19 plasma, freshly isolate neutrophils were plated on

81196 well plates (Corning, 3595) coated with 0.01\% poly-L-lysine (Sigma, P4707) in IMDM

812 supplemented with $0.5 \%$ hiFBS. The neutrophils were allowed to settle and adhere for $20 \mathrm{~min}$

813 before the media was removed and IMDM containing 10\% healthy or COVID-19 plasma and 814 with or without glycopolypeptide $(500 \mathrm{nM})$ was added. Alternatively, control wells were treated 815 with no inducer, PMA (500 nM) or R848 (10 $\mu \mathrm{M})$ in IMDM with $0.5 \%$ hiFBS. Plates were 816 incubated for $4 \mathrm{~h}$ at $37^{\circ} \mathrm{C}$ and the media was gently removed. The cells were then fixed for 15 $817 \mathrm{~min}$ at room temperature in a solution of 4\% PFA in PBS (ThermoFisher, FB002). The fixed 
822

823

824

825

826

827

828

cells were then blocked in 10\% goat serum in PBS, stained with anti-H1/DNA (EMD Millipore, MAB3864), and visualized with anti-mouse AlexaFluor 594 (Jackson ImmunoResearch, 115585-174). Plates were imaged using an Incucyte S3.

For NETosis assays with undiluted COVID-19 plasma, freshly isolate neutrophils were plated on black-walled half-area 96 well plates (Greiner, 675090) coated with 0.01\% poly-L-lysine (Sigma, P4707) in IMDM supplemented with $0.5 \%$ hiFBS. The neutrophils were allowed to settle and adhere for 20 min before the media was removed and either healthy or COVID-19 plasma and with or without glycopolypeptide (500 nM) was added. Alternatively, control wells were treated with no inducer or R848 $(10 \mu \mathrm{M})$ in IMDM with $0.5 \%$ hiFBS. Plates were incubated for $4 \mathrm{~h}$ at 37 ${ }^{\circ} \mathrm{C}$ and the media was gently removed. The cells were then fixed for $15 \mathrm{~min}$ at room temperature in a solution of 4\% PFA in PBS (ThermoFisher, FB002). The fixed cells were washed. Then cells were then blocked in 10\% goat serum. For plates imaged by Incucyte S3, cells were stained with anti-H1/DNA (EMD Millipore, MAB3864) and visualized with a combination of anti-mouse AlexaFluor 594 (Jackson ImmunoResearch, 115-585-174) and MemGlow488 (Cytoskeleton, MG01-02). For plates imaged by immunofluorescence using a Keyence BZ-710, cells were stained with anti-MPO (Thermo Scientific, MA516383) and visualized with anti-rabbit AlexaFluor 555 (Thermo Scientific, A27039). Cells were imaged in HBSS containing DAPI imaging solution (Thermo Scientific, R37606). Images were collected using a Keyence BZ-X710 and images were analyzed using ImageJ.

Incucyte images were analyzed using the onboard Incucyte analysis software. For Incucyte S3 analyses: phase images were analyzed to identify cells using the following settings: segmentation adjustment $=0.8$; minimum area filter $=25 \mu \mathrm{m}^{2}$. For primary neutrophils, 
842 fluorescence areas were determined using the following settings: top hat segmentation; radius

$843(\mu \mathrm{m})=10.0$; threshold $=10.0 \mathrm{GCU}$ or $0.1 \mathrm{RCU}$; area filter $=300 \mu \mathrm{m}^{2}$. For $\mathrm{dHL}-60 \mathrm{~s}$,

844 fluorescence areas were determined as for primary neutrophils with the following variation: area

845 filter $=200 \mu \mathrm{m}^{2}$. For Incucyte ZOOM analyses: phase images were analyzed to identify cells

846 using the following settings: segmentation adjustment $=0.8$; minimum area filter $=25 \mu m^{2}$. For

847 primary neutrophils, fluorescence areas were determined using the following settings: top hat

848 segmentation; radius $(\mu \mathrm{m})=10.0$; threshold $=5.0 \mathrm{GCU}$; area filter $=200 \mu \mathrm{m}^{2}$

Reactive oxygen species measurements.

851 Induction of reactive oxygen species was measured by fluorescence microscopy using an

852 Incucyte S3 (Essen Biosciences). Cells were prepared as for NETosis assays, with the

853 difference that rather than the inclusion of Cytotox dye in the media, CellROX Deep Red

854 (Thermo Fisher, C10422) was included at a final concentration of $5 \mu \mathrm{M}$. Data were analyzed as 855 in NETosis assays, with the following changes in settings for quantifying red fluorescence: top 856 hat segmentation; radius $(\mu \mathrm{m})=10.0$; threshold $=1.6 \mathrm{RCU}$; area filter $=25 \mu \mathrm{m}^{2}$.

858 siRNA knock downs.

859 For siRNA knock down experiments, HL-60's were differentiated as indicated above. On day 4, 860 the media was changed and $3 \mathrm{~mL}$ of culture was transfected with 30 pmol siRNA's (IDT) using 861 Lipofectamine RNAiMAX (Thermo Fisher, 13778100) according to the manufacturer's 862 instructions. Transfectants were cultured for $48 \mathrm{~h}$ prior to use in NETosis assays, with a 863 complete media change at $24 \mathrm{~h}$ post transfection. Protein expression was validated by Western 864 blot or flow cytometry, as applicable. 
866 For siRNA KD of SIGLEC9, the top two suggested predesigned dicer substrate siRNA's from

867 IDT (hs.Ri.SIGLEC9.13.1 and hs.Ri.SIGLEC9.13.2) were combined in a 1:1 molar ratio. For

868 siRNA KD of PTPN6, the top suggested predesigned dicer substrate siRNA (hs.Ri.PTPN6.13.1)

869 was used. As a negative control, scramble siRNA's were obtained from IDT.

871 Flow cytometry.

872 All flow cytometry experiments were performed using a BD Accuri 6 flow cytometer and 873 analyzed using FlowJo 10. For all flow cytometry experiments, live cells were isolated from 874 culture and resuspended in cold PBS supplemented with 1\% BSA at a concentration of 1 e7 875 cells per $\mathrm{mL}$. Aliquots of $5 \mathrm{e} 5$ cells were then stained at the concentrations indicated in the 876 Reagents Table and Usage for $30 \mathrm{~min}$ on ice in the dark. Cells were then washed three times 877 (500 rcf, $5 \mathrm{~min}$ ) with in cold PBS supplemented with 1\% BSA before being analyzed by flow 878 cytometry. SDS-PAGE and Western blotting.

881 Cell pellets were washed twice with cold PBS supplemented with 1 mM EDTA and were lysed in 882 RIPA buffer (50 $\mu \mathrm{L}$ for each 1 e6 cells) (Thermo Fisher, PI89900) supplemented with Benzonase 883 (1:1000) (Sigma Aldrich, E1014), HALT phosphatase inhibitor (1:100) (Thermo Fisher, 78420), 884 and HALT protease inhibitor (1:100) (Thermo Fisher, 87786) for 30 min at $4^{\circ} \mathrm{C}$. Lysates were 885 clarified by centrifugation at $4{ }^{\circ} \mathrm{C}$ for $15 \mathrm{~min}$ at 16000 rcf. Protein concentration was quantified 886 by Rapid Gold BCA (Thermo Fisher, A53226). 
Lysates were diluted into SDS-buffer with beta-mercaptoethanol and were separated by SDS-

889 PAGE on 4-12\% bisacrylamide gels loading $10 \mu \mathrm{g}$ per lane. For analysis of histone H3

890 citrullination, lysates were separated in XT-MES at $200 \mathrm{~V}$ for $35 \mathrm{~min}$. For analysis of SHP-1

891 levels after siRNA KD, lysates were separated in XT-MOPS at $200 \mathrm{~V}$ for $1 \mathrm{~h}$. Blots were

892 transferred to nitrocellulose and blocked with 5\% BSA in TBS before being stained with primary

893 antibodies and IR-dye conjugated secondary antibodies for analysis by LiCOR.

\section{Protein expression and purification.}

896 All proteins for enzymatic synthesis of glycopolypeptides were expressed and purified as 897 previously described..$^{41}$

898 His-tagged proteins cultures were grown at $37^{\circ} \mathrm{C}$ to an OD600 of $0.8-1.0$ in $1 \mathrm{~L}$ of LB containing 899 the appropriate antibiotic selection marker, at which point expression was induced with IPTG $900(0.1 \mathrm{mM})$ and cultures were grown overnight at $20^{\circ} \mathrm{C}$ with shaking at $220 \mathrm{rpm}$. After $24 \mathrm{~h}$, cells 901 were pelleted by centrifugation and lysed in buffer $(250 \mathrm{mM}$ Tris $\mathrm{HCl}, 0.5 \mathrm{M} \mathrm{NaCl}, 20 \mathrm{mM}$ 902 imidazole, $0.1 \%$ TritonX100) supplemented with protease inhibitor cocktail (Sigma Aldrich, 903 04693132001) (one tablet per $40 \mathrm{~mL}$ ) and DNAsel (Thermo Fisher, 90083) (10 $\mu \mathrm{L}$ per $40 \mathrm{~mL}$ ). 904 Cells were lysed using a dounce homogenizer followed by French press. Lysates were clarified 905 by centrifugation and purified on HisTRAP columns (GE Life Sciences, 17-5247-01) using a 906 gradient of $20 \mathrm{mM}$ to $200 \mathrm{mM}$ imidazole on an AKTA FPLC. Fraction purity was determined by 907 SDS-PAGE and pure fractions were combined, purified by dialysis against storage buffer (50 $908 \mathrm{mM}$ Tris $\mathrm{HCl}, 250 \mathrm{mM} \mathrm{NaCl}, 10 \%$ glycerol), aliquoted, and flash-frozen in liquid nitrogen for 909 storage in $\mathrm{a}-80^{\circ} \mathrm{C}$ freezer. 
912 Primary neutrophils were treated in media containing the indicated compounds in IMDM [+]

$913 \quad 0.5 \%$ hiFBS for the indicated time period, or in plasma from either healthy donors or COVID-19

914 patients, with or without pS9L $(500 \mathrm{nM})$ for $15 \mathrm{~min}$. Cell pellets were washed twice with cold

915 PBS supplemented with $1 \mathrm{mM}$ EDTA and were lysed in RIPA buffer (50 uL for each 1 e6 cells)

916 (Thermo Fisher, PI89900) supplemented with Benzonase (1:1000) (Sigma Aldrich, E1014),

917 HALT phosphatase inhibitor (1:100) (Thermo Fisher, 78420), and HALT protease inhibitor

918 (1:100) (Thermo Fisher, 87786) for $30 \mathrm{~min}$ at $4{ }^{\circ} \mathrm{C}$. Lysates were clarified by centrifugation at 4

$919{ }^{\circ} \mathrm{C}$ for 15 min at 16000 rcf. Protein concentration was quantified by Rapid Gold BCA (Thermo

920 Fisher, A53226).

921 Digestion was performed on $100 \mu \mathrm{g}$ protein using a mini S-trap protocol provided by the 922 manufacturer (Protifi). ${ }^{67}$ Here, proteins brought to 5\% SDS and reduced with 5 mM DTT for 10 923 minutes at $95 \mathrm{C}$. Cysteines were alkylated using $30 \mathrm{mM}$ iodoacetamide for 45 minutes each at 924 room temperature in the dark. The lysate was then acidified with phosphoric acid, brought to 925 approximately $80-90 \%$ methanol with 100 mM TEAB in $90 \%$ methanol, and loaded onto the S926 trap column. Following washing with 100 mM TEAB in 90\% methanol, trypsin (Promega) was 927 added to the S-trap at a 20:1 protein:protease ratio for 90 minutes at $47^{\circ} \mathrm{C}$. Peptides from each 928 lysate were labeled with 11-plex TMT (Tandem Mass Tags, Thermo Fisher Scientific) for 2 929 hours at room temperature using recently published protocols. ${ }^{47,68}$ Labeling schemes for the 930 stimulated study comparing R848 and PMA to no treatment (NT) were: NT replicates in 931 channels 126C (Donor 1), 127N (Donor 2), and 130C (Donor 3); PMA replicates in 127C (Donor 932 1), 128N (Donor 2) and 131N (Donor 3); R848 replicates in 128C (Donor 1), 129N (Donor 2) 933 and 131C (Donor 3). For the polymer experiment, the labeling scheme was: vehicle replicates in 934 channels 126C (Donor 1), 127N (Donor 2), and 130C (Donor 3); pS9L replicates in 127C (Donor 935 1), 128N (Donor 2) and 131N (Donor 3); pLac replicates in 128C (Donor 1), 129N (Donor 2) and $936131 \mathrm{C}$ (Donor 3). A test mix was run to confirm $>99 \%$ labeling efficiency and even distribution of 
937 signal across all channels prior to quenching of the TMT labeling reaction $(0.5 \mathrm{uL} 50 \%$ hydroxylamine reacted for $15 \mathrm{~min}$ ). Peptides from each channel were then combined prior to phosphopeptide enrichment, which was performed as previously described. ${ }^{69}$ Briefly, $100 \mu \mathrm{L}$ magnetic titanium(IV) immobilized metal ion affinity chromatography (Ti(IV)-IMAC, ReSyn Biosciences) beads were washed three times with $1 \mathrm{~mL} 80 \%$ acetonitrile/6\% TFA (all washes were $1 \mathrm{~mL}) .{ }^{70}$ Peptides were dissolved in $1 \mathrm{~mL} 80 \%$ acetonitrile/6\% TFA and gently vortexed with the $\mathrm{TI}(\mathrm{IV})$-IMAC beads for 45 minutes. Unbound peptides were kept as flow through for total protein analysis, followed by three $80 \%$ acetonitrile/6\% TFA, one $80 \%$ acetonitrile, one 0.5 M glycolic acid/80\% acetonitrile, and two $80 \%$ acetonitrile washes. Peptides were eluted with $500 \mu \mathrm{L} \mathrm{50 \%}$ acetonitrile, $1 \%$ ammonium hydroxide. Both eluate and flow through were dried down in a speed vac and further cleaned up on Strata-X SPE cartridges (Phenomenex) by conditioning the cartridge with $1 \mathrm{~mL} A C N$ followed by $1 \mathrm{~mL} 0.2 \%$ formic acid (FA) in water. Peptides were resuspended in $0.2 \%$ FA in water and then loaded on to the cartridge, followed by a $1 \mathrm{~mL}$ wash with $0.2 \% \mathrm{FA}$ in water. Peptides were eluted with $400 \mathrm{uL}$ of $0.2 \% \mathrm{FA}$ in $80 \%$ ACN, were dried via lyophilization.

All samples were resuspended in $0.2 \%$ formic acid in water prior to LC-MS/MS analysis. Total protein samples were resuspended in $500 \mu \mathrm{L}$ with $1 \mu \mathrm{L}$ injected on column, while enriched phosphopeptides were resuspended in $15 \mu \mathrm{L}$ total with $4 \mu \mathrm{L}$ injected per analysis. Triplicate injections were collected for all samples. All (phospho)peptide mixtures were separated over a $25 \mathrm{~cm}$ EasySpray reversed phase LC column (75 $\mu \mathrm{m}$ inner diameter packed with $2 \mu \mathrm{m}, 100 \AA$, PepMap C18 particles, Thermo Fisher Scientific). The mobile phases (A: water with $0.2 \%$ formic acid and B: acetonitrile with $0.2 \%$ formic acid) were driven and controlled by a Dionex Ultimate 3000 RPLC nano system (Thermo Fisher Scientific). An integrated loading pump was used to load peptides onto a trap column (Acclaim PepMap 100 C18, 5 um particles, $20 \mathrm{~mm}$ length, 
962 Thermo Fisher Scientific) at $8 \mu \mathrm{L} / \mathrm{min}$, which was put in line with the analytical column 4 minutes

963 into the gradient for the total protein samples. The gradient increased from $0 \%$ to $5 \% \mathrm{~B}$ over the

964 first 4 minutes of the analysis, followed by an increase from $5 \%$ to $25 \%$ B from 4 to 158 minutes, 965 an increase from $25 \%$ to $90 \%$ B from 158 to 162 minutes, isocratic flow at $90 \%$ B from 162 to

966168 minutes, and a re-equilibration at $0 \%$ for 12 minutes for a total analysis time of 180 minutes.

967 Eluted (phoshpo)peptides were analyzed on an Orbitrap Fusion Tribrid MS system (Thermo

968 Fisher Scientific). Precursors were ionized using an EASY-Spray ionization source (Thermo

969 Fisher Scientific) source held at $+2.2 \mathrm{kV}$ compared to ground, and the column was held at 40

$970{ }^{\circ} \mathrm{C}$. The inlet capillary temperature was held at $275^{\circ} \mathrm{C}$. Survey scans of peptide precursors were

971 collected in the Orbitrap from 350-1350 Th with an AGC target of 1,000,000, a maximum

972 injection time of $50 \mathrm{~ms}$, and a resolution of 60,000 at $200 \mathrm{~m} / \mathrm{z}$. Monoisotopic precursor selection

973 was enabled for peptide isotopic distributions, precursors of $z=2-5$ were selected for data-

974 dependent MS/MS scans for 2 second of cycle time, and dynamic exclusion was set to 30

975 seconds with a \pm 10 ppm window set around the precursor monoisotope. An isolation window of

9761 Th was used to select precursor ions with the quadrupole. MS/MS scans were collected using

977 HCD at 30 normalized collision energy (nce) with an AGC target of 100,000 and a maximum

978 injection time of $118 \mathrm{~ms}$. Mass analysis was performed in the Orbitrap with a resolution of

97960,000 with a first mass set at 100 Th.

981 Phosphoproteomic data analysis.

982 All data were searched with the Andromeda search engine ${ }^{71}$ in MaxQuant ${ }^{72}$ using the entire 983 human proteome downloaded from Uniprot $^{73}$ (reviewed, 20428 entries). Each separate TMT 984 experiment (resting, activated, and pLac control) was searched separately, with the flow 985 through/total protein triplicate injections labeled as Group0 and False under "PTM" and 986 phosphopeptide enriched triplicate injections labeled as Group1 and True under "PTM". Group0 
had cleavage specificity set to Trypsin/P with 2 missed cleavage allowed and variable modifications of oxidation of methionine and acetylation of the protein $\mathrm{N}$-terminus with 4 maximum modifications per peptide. Group1 had cleavage specificity set to Trypsin/P with 3

990 missed cleavage allowed and variable modifications of phosphorylation on

991 serine/threonine/tyrosine, oxidation of methionine, and acetylation of the protein $\mathrm{N}$-terminus with 9924 maximum modifications per peptide. The experiment type for both Group0 and Group1 was 993 set to Reporter ion MS2 and only TMT channels used (as described above) were selected to be 994 included. The reporter ion mass tolerance was set to 0.3 $\mathrm{Da}$ and the minimum reporter PIF 995 score was set to 0.75. Defaults were used for the remaining settings, including PSM and protein 996 FDR thresholds of 0.01 and 20 ppm, 4.5 ppm, and 20 ppm for first search MS1 tolerance, main 997 search MS1 tolerance, and MS2 product ion tolerance, respectively. Match between runs was 998 not enabled. Quantified phosphosites were then processed in Perseus. ${ }^{74}$ Contaminants and 999 reverse hits were removed, results were filtered for phosphosites that had localization 1000 probabilities $>0.75$, and signal in all relevant TMT channels was required. Significance testing 1001 was performed using a two-tailed pair-ed sample t-test calculated in Microsoft Excel, using one 1002 condition versus control (NT or vehicle for stimulated and polymer experiments, respectively) for 1003 pairwise comparisons. Data have been deposited to the ProteomeXchange Consortium via the 1004 PRIDE partner repository with the dataset identifier PXD022990. ${ }^{75}$

\section{Analysis of publicly-available single-cell RNA-sequencing (scRNA-seq) data.}

1007 The open source statistical software R (www.r-project.org; v3.6.1) and the R package Seurat (v 1008 3.2.2) was used for scRNA-seq data analysis. ${ }^{76}$ A pre-processed Seurat object containing 1009 scRNA-seq data and metadata of neutrophils profiled using the BD Rhapsody platform was 1010 retrieved from www.fastgenomics.org as outlined in the data availability statement of Schulte1011 Schrepping, et al. ${ }^{8}$ The average expression of SIGLEC9 or PADI4 was defined as the mean of 
1012 log-normalized transcript counts, calculated by NormalizeData() function, in a given sample.

1013 DotPlot() was used to visualize average and percent expression of SIGLEC9 or PADI4.

Reagent Table and Usage.

1016 IN - Incucyte S3 (microscopy); OC - Octet (in vitro protein binding); FC - flow cytometry; WB -

1017 western blot; KD - siRNA knock down; IF - immunofluorescence

\begin{tabular}{|l|l|l|}
\hline Reagent & Source (\#) & Usage, dilution/concentration \\
\hline Cytotox Green & Essen Biosciences (4633) & IN, 1:4,000 \\
\hline Cytotox Red & Essen Biosciences (4632) & IN, 1:4,000 \\
\hline CellROX Deep Red & ThermoFisher (C10422) & IN, 1:500 \\
\hline $\begin{array}{l}\text { Anti-Siglec-9 clone K8 / } \\
\text { AlexaFluor 647 }\end{array}$ & BioLegend (351509) & FC, 1:50 \\
\hline $\begin{array}{l}\text { Anti-human CD45 clone } \\
\text { HI30 / APC }\end{array}$ & $\begin{array}{l}\text { Stemcell Tecnologies } \\
\text { (60018AZ.1) }\end{array}$ & FC, 1:50 \\
\hline $\begin{array}{l}\text { Anti-human CD16 clone } \\
\text { 3G8 / AlexaFluor 488 }\end{array}$ & $\begin{array}{l}\text { Stemcell Technologies } \\
\text { (60041AD.1) }\end{array}$ & FC, 1:50 \\
\hline $\begin{array}{l}\text { Mouse IgG1 isotype clone } \\
\text { MOPC-21 / FITC }\end{array}$ & BD Biosciences (551954) & FC, 1:50 \\
\hline $\begin{array}{l}\text { Mouse IgG1 isotype clone } \\
\text { MOPC-21 / APC }\end{array}$ & BD Biosciences (550854) & FC, 1:50 \\
\hline Siglec-9-Fc & R\&D Systems (1139-SL-050) & OC, 400 nM \\
\hline DsiRNA (SIGLEC9) & IDT (hs.Ri.SIGLEC9.13.1) & KD, 30 nM \\
\hline DsiRNA (SIGLEC9) & IDT (hs.Ri.SIGLEC9.13.2) & KD, 30 nM \\
\hline DsiRNA (PTPN6) & IDT (hs.Ri.PTPN6.13.1) & KD, 30 nM \\
\hline siRNA negative control & IDT (51-01-19-08) & KD, 30 nM \\
\hline $\begin{array}{l}\text { Rabbit anti-CitH3 } \\
\text { (R2/R8/R17) }\end{array}$ & Abcam (ab5103) & WB, 1:1,000 \\
\hline Mouse anti-GAPDH & $\begin{array}{l}\text { Sigma-Aldrich (G8795- } \\
100 U L) ~\end{array}$ & WB, 1:10,000 \\
\hline NSC-87877 & $\begin{array}{l}\text { Sigma-Aldrich (565851- } \\
50 M G) ~\end{array}$ & IN, 50 $\mu M$ \\
\hline
\end{tabular}




\begin{tabular}{|l|l|l|}
\hline $\begin{array}{l}\text { Rabbit anti-SHP1 (clone } \\
\text { Y476) }\end{array}$ & Abcam (ab32559) & WB, 1:1,000 \\
\hline goat anti-mouse 680RD & LiCOR (926-68070) & WB, 1:10,000 \\
\hline goat anti-rabbit 800CW & LiCOR (926-32211) & WB, 1:10,000 \\
\hline anti-H1/DNA & EMD Millipore (MAB3864) & IF, 1:100 \\
\hline $\begin{array}{l}\text { goat anti-mouse } \\
\text { AlexaFluor 594 }\end{array}$ & $\begin{array}{l}\text { Jackson ImmunoResearch } \\
(115-585-174)\end{array}$ & IF, 1:1000 \\
\hline MemGlow488 & Cytoskeleton (MG01-02) & IF, 1:200 \\
\hline $\begin{array}{l}\text { anti-myeloperoxidase } \\
\text { clone SP72 }\end{array}$ & $\begin{array}{l}\text { Thermo Scientific } \\
\text { (MA516383) }\end{array}$ & IF, 1:100 \\
\hline $\begin{array}{l}\text { goat anti-rabbit AlexaFluor } \\
\text { 555 }\end{array}$ & Thermo Scientific (A27039) & IF, 1:1000 \\
\hline DAPI solution & Thermo Scientific (R37606) & IF, 2 drops per mL \\
\hline
\end{tabular}

1018 